\title{
An Evolutionary Algorithm for Multiobjective Fuzzy Portfolio Selection Models with Transaction Cost and Liquidity
}

\author{
Wei Yue, Yuping Wang, and Cai Dai \\ School of Computer Science and Technology, Xidian University, Xian, Shaanxi 710071, China \\ Correspondence should be addressed to Wei Yue; yuewei@stu.xidian.edu.cn
}

Received 6 April 2015; Revised 14 June 2015; Accepted 1 July 2015

Academic Editor: Yang Tang

Copyright ( 2015 Wei Yue et al. This is an open access article distributed under the Creative Commons Attribution License, which permits unrestricted use, distribution, and reproduction in any medium, provided the original work is properly cited.

\begin{abstract}
The major issues for mean-variance-skewness models are the errors in estimations that cause corner solutions and low diversity in the portfolio. In this paper, a multiobjective fuzzy portfolio selection model with transaction cost and liquidity is proposed to maintain the diversity of portfolio. In addition, we have designed a multiobjective evolutionary algorithm based on decomposition of the objective space to maintain the diversity of obtained solutions. The algorithm is used to obtain a set of Pareto-optimal portfolios with good diversity and convergence. To demonstrate the effectiveness of the proposed model and algorithm, the performance of the proposed algorithm is compared with the classic MOEA/D and NSGA-II through some numerical examples based on the data of the Shanghai Stock Exchange Market. Simulation results show that our proposed algorithm is able to obtain better diversity and more evenly distributed Pareto front than the other two algorithms and the proposed model can maintain quite well the diversity of portfolio. The purpose of this paper is to deal with portfolio problems in the weighted possibilistic mean-variance-skewness (MVS) and possibilistic mean-variance-skewness-entropy (MVS-E) frameworks with transaction cost and liquidity and to provide different Pareto-optimal investment strategies as diversified as possible for investors at a time, rather than one strategy for investors at a time.
\end{abstract}

\section{Introduction}

The portfolio selection problem is an important issue in the theory and practice of finance. Modern portfolio selection theory originated from the pioneering research work of Markowitz's mean-variance model [1]. The mean-variance model is a bicriteria optimization problem where a rational portfolio choice is based on trade-off between risk and return. However, numerous authors, such as Leland [2], Kerstens et al. [3], and Arditti and Levy [4], pointed out that mean-variance portfolio theory is not consistent with the Von Neumann-Morgenstern axioms of expected utility theory unless either asset processes have normal probability distributions or the utility functions of investor preferences are quadratic. Indeed, many academic researchers, such as Lai [5], Adcock and Shutes [6], Narayan and Ahmed [7], and Engle and Mistry [8], revealed that portfolio returns are not, in general, normally distributed and the asset distributions are often positively skewed. Furthermore, starting from Pindoriya et al. [9], Eichner and Wagener [10], and Yu et al. [11], among others, a multitude of studies showed that investors prefer positive skewness, which means that utility functions of investors are not quadratic. As a result, the concept of mean-variance trade-off has been extended to include the skewness of return in portfolio selection. In other words, the mean-variance-skewness trade-off model for portfolio selection has been generated.

All the above studies assumed that the returns of risk assets are random variables with some probability distributions. However, as well known, the uncertain returns of risk assets are influenced by economic change, politics, social conditions, the status of the related company, and so forth. In addition, security markets are usually very sensitive. An accident or a hard-to-verify message may influence security prices or returns greatly. Thus, in reality, the uncertain security returns are hard to get because of the complexity of the financial markets and many unexpected factors, and the estimation of uncertain security returns mainly relies on expert's evaluations. Obviously, in many situations, it is more suitable to estimate the returns of risk assets by using 
fuzzy variables. In fact, with the development of security market and fuzzy set theory, more and more attention has been paid to fuzzy portfolio selection models. Recently, some researchers extended the mean-variance model to a meanvariance-skewness framework in fuzzy environment. For example, Bhattacharyya et al. [12] considered fuzzy meanvariance-skewness portfolio selection by using the concept of interval valued fuzzy numbers and presented a hybrid intelligence algorithm to solve the model. Barak et al. [13] proposed a fuzzy portfolio mean-variance-skewness model with cardinality constraint which combined assets limitations with liquidity requirement. Liu et al. [14] proposed a multiperiod portfolio selection problem in fuzzy environment by considering return, risk, skewness, and transaction cost and presented a TOPSIS-compromised programming approach to convert them into single-objective programming models to resolve them. More recently, fuzzy portfolio selection problem is one of the hottest research areas in the field of finance.

It is worth pointing out that although the great progress has been made in the previous studies, there are still certain limitations. One of the major issues for portfolio models in mean-variance-skewness framework is low diversity in the portfolio or the corner solutions. The low diversity of the portfolio may result in loss while some of the invested assets experience unexpected gains and make the performance of the portfolio models not very good $[15,16]$. Therefore, to assess diversification, the entropy is used as established measure of diversity in the literature (e.g., see [17-19] and the references therein). In this paper, we maximize the entropy function to generate a well-diversified portfolio. Secondly, the mean-variance-skewness trade-off model for portfolio selection is not easy to find a trade-off between the three objectives because this is a nonlinear and nonsmooth multiobjective optimization problem. Until now, many methods are used to tackle such problems that have been restricted to goal programming or multicriterion decision-making methods, which suggests converting the multiobjective optimization problem to a single-objective optimization problem by emphasizing one particular Pareto-optimal solution at a time. When such methods are used for finding multiple solutions, they have to be applied many times, hopefully finding a different solution in each simulation run. To overcome these limitations, we design a multiobjective evolutionary algorithm based on decomposition of the objective space to solve the problems. Our algorithm is able to find more diversity and multiple Pareto-optimal solutions in one single simulation run. Thirdly, most of existing portfolio selection models have focused on only two fundamental factors, that is, expected return and risk of portfolio; however, in realistic investment, the transaction cost is an important factor for investors, which cannot be ignored. It is well known that ignoring transaction costs would result in an inefficient portfolio and adding transaction costs would assist decision makers to better understand the behavior of an efficient frontier. On the other hand, the liquidity is also another major concern of portfolio selections, and most of shortterm investors are willing to own the assets that can be sold easily. What is more, exchange market authorities and brokers profit from high turnover rate, because they can gain more fees by high traded asset volume, instead of asset price change. That is why they are also willing to facilitate trading these assets more than other ones. The liquidity is usually measured by using turnover rate of asset. So, turnover rate is a vital criterion for investors. Owing to the importance of asset liquidity and owing to the preference of investors for the liquidity of their portfolio, we consider the turnover rate standards in our proposed models. Motivated by these considerations, we use the entropy as an objective function to generate a well-diversified portfolio and take into account the liquidity and transaction cost on portfolio to make the model more practical; in addition, we propose a new evolutionary algorithm for the nonlinear and nonsmooth multiobjective problem.

To our best knowledge, for the first time, the multiobjective mean-variance-skewness-entropy portfolio model with transaction cost and liquidity is proposed in fuzzy environment, and the multiobjective evolutionary algorithm is presented to deal with such a complicated problem. In our work, to obtain a well-diversified portfolio, the entropy is used as an objective function in the proposed model; furthermore, a new evolutionary algorithm based on decomposition of the objective space is designed to achieve the goal.

On the other hand, from the viewpoint of optimization, a single solution that simultaneously optimizes all the conflicting objectives hardly exists in practice. Instead, there exists a set of acceptable efficient solutions (largely known as Pareto-optimal solutions or nondominated solutions) which are optimal in such a way that no other solutions are superior to them when all objectives are considered simultaneously. So, this demands us to find Pareto-optimal portfolios as many as possible. Among various multiobjective optimization algorithms, multiobjective evolutionary algorithms (MOEAs), which make use of the strategy of the population evolution to optimize the problems, are effective methods for solving multiobjective optimization problems (MOPs). In recent years, many MOEAs, such as NSGA-II [20], MOEA/D [21], FWEA [22], and p-OCEA [23], have been proposed for solving the multiobjective optimization problems. In comparison with other traditional optimization algorithms, the multiobjective evolutionary algorithms (MOEAs) aim at finding a set of representative Pareto-optimal solutions in a single run. MOEAs have attracted a lot of research effort during the last 20 years, and they are still the hottest research areas in the field of evolutionary computations.

In this paper, the portfolio models are not differentiable, nonlinear, and nonsmooth multiobjective optimization problems which are very challenging and intractable optimization problems. To solve these complicated tasks, we design a new multiobjective evolutionary algorithm [24] based on decomposition of the objective space which can well maintain the diversity of obtained solutions. To achieve the goal, we decompose the objective space of the MOPs into a set of subjective spaces by a set of direction vectors. Each subjective space has a solution: if a new solution will replace the solution, the new solution must dominate the solution and its objective vector is located in the subjective space. In this way, the diversity of obtained solutions can be maintained, which 
is vital for solving the MOPs. Furthermore, by using the crowding distance [20], the fitness value of a solution is calculated for the selection operators. In this case, when a solution dominates other solutions, it is more possible to be chosen than other solutions, and then more new solutions can be generated so as to quickly seek out the optimal solution of the subobjective space, which makes the solution to each subobjective space converge to the optimal solutions as far as possible. By these approaches, a new multiobjective evolutionary algorithm is proposed.

The rest of this paper is organized as follows. Section 2 introduces basic definitions and preliminary results related to fuzzy variables. In Section 3, we propose the weighted possibilistic MVS and MVS-E multiobjective portfolio models with transaction cost and liquidity. Section 4 presents a detailed description of our proposed multiobjective evolution algorithm. Section 5 shows the comparisons of our algorithm with MOEA/D, NSGA-II, and D2MOPSO and gives the result analysis between weighted MVS and MVS-E models. The last section gives some concluding remarks.

\section{Basic Concepts and Definitions}

We first introduce some concepts and results about fuzzy number, which will be needed in the following sections.

2.1. On Fuzzy Numbers and Notations. In this section, the basic concepts and notations are given as follows $[24,25]$.

Definition 1. A fuzzy number $\widetilde{A}$ is expressed as any fuzzy subset of the real line $\mathbb{R}$, whose membership function $\mu_{\widetilde{A}}$ : $\mathbb{R} \rightarrow[0,1]$ satisfies the following conditions:

(i) $\widetilde{A}$ is normal; that is, there exists $x \in \mathbb{R}$ such that $\mu_{\widetilde{A}}(x)=1$;

(ii) $\mu_{\widetilde{A}}(x)$ is quasi-concave; that is, $\mu_{\widetilde{A}}(\lambda x+(1-\lambda) y) \leq$ $\min \left\{\mu_{\widetilde{A}}(x), \mu_{\widetilde{A}}(y)\right\}$, for all $\lambda \in[0,1]$;

(iii) $\mu_{\widetilde{A}}(x)$ is upper semicontinuous; that is, $\left\{x \in \mathbb{R} \mid \mu_{\widetilde{A}} \leq\right.$ $\varepsilon\}$ is a closed set, for all $\varepsilon \in[0,1]$;

(iv) the closure of the set $\left\{x \in \mathbb{R} \mid \mu_{\widetilde{A}}>0\right\}$ is a compact set.

Definition 2. A $\gamma$-level set of $\widetilde{A}$ is defined by $\widetilde{A}_{\gamma}=\{x \in \mathbb{R} \mid$ $\left.\mu_{\widetilde{A}}(x) \geq \gamma\right\}$ if $\gamma>0$ and $\widetilde{A}_{\gamma}=\operatorname{cl}\left\{x \in \mathbb{R} \mid \mu_{\widetilde{A}}(x) \geq 0\right\}$ (the closure of the support of $\widetilde{A}$ ) if $\gamma=0$. As well known, if $\widetilde{A}$ is a fuzzy number, then $\widetilde{A}_{\gamma}=\left\{x \in \mathbb{R} \mid \mu_{\widetilde{A}} \geq \gamma\right\}=[\underline{a}(\gamma), \bar{a}(\gamma)]$ is a compact subset of $\mathbb{R}$ for all $\gamma \in[0,1]$.

Definition 3. A fuzzy number $\widetilde{A}$ is called a trapezoidal fuzzy number with core $[c, d]$, left width $\delta$, and right width $\theta$ if its membership function has the following form:

$$
\mu_{\widetilde{A}}(x)= \begin{cases}1-\frac{c-x}{\delta}, & \text { if } c-\delta \leq x \leq c \\ 1, & \text { if } c \leq x \leq d \\ 1-\frac{x-d}{\theta}, & \text { if } d \leq x \leq d+\theta \\ 0, & \text { if otherwise }\end{cases}
$$

and it can be represented as the notation $\widetilde{A}=(c, d, \delta, \theta)$.

2.2. Weighted Possibilistic Moments (WPMs). In this paper, we regard a new weighted possibilistic mean value, variance, and skewness [26] of fuzzy return to characterize the return level, risk level, and the corresponding asymmetry as alternative approach, respectively. It is just because the weighted possibilistic mean (WPM) and variance (WPV) of fuzzy number have all the properties of the possibilistic mean value and variance stated in $[24,25]$, and the WPV has all necessities and important properties for defining of the possibilistic variance of a fuzzy number. In addition, WPM is the nearest weighted point to the fuzzy number via minimizing a new weighted distance quantity; moreover, WPV of a fuzzy number is consistent with the physical interpretation of the variance and well-known definition of variance in probability theory so that it can simply introduce the possibilistic moments about the mean of fuzzy numbers [26]. Furthermore, Pasha et al. [26] pointed out that this definition of weighted possibilistic moments on fuzzy number is more suitable for all fuzzy numbers than the definitions of possibilistic moments introduced in $[24,25]$. This indicates that WPM and WPV are suitable and applicable and play an important role in fuzzy data analysis. For this reason, we quantify the return, risk, and skewness by using the WPMs.

The definitions of the possibilistic moments of fuzzy numbers are obtained from [26].

Definition 4. Let $\widetilde{A}$ be a fuzzy number with $\widetilde{A}_{\gamma}=$ $[\underline{a}(\gamma), \bar{a}(\gamma)], \gamma \in[0,1]$. Then the WPM (or the first order $f$ WPM) and WPV of fuzzy number $\widetilde{A}$ are defined as follows, respectively:

(i) $M_{f}(\widetilde{A})=\int_{0}^{1} f(\gamma)((\underline{a}(\gamma)+\bar{a}(\gamma)) / 2) d \gamma$;

(ii) $\operatorname{Var}_{f}(\widetilde{A})=(1 / 2) \int_{0}^{1} f(\gamma)\left[\left(\underline{a}(\gamma)-M_{f}(\widetilde{A})\right)^{2}+(\bar{a}(\gamma)-\right.$ $\left.\left.M_{f}(\widetilde{A})\right)^{2}\right] d \gamma$,

where $f(\gamma)=(n+1) \gamma^{n}$ is a weighted function such that $\int_{0}^{1} f(\gamma) d \gamma=1$.

Definition 5. Let $\widetilde{A}$ and $\widetilde{B}$ be two fuzzy numbers with weighted possibilistic mean $M_{f}(\widetilde{A})$ and $M_{f}(\widetilde{B})$, respectively. Then the $f$-weighted possibilistic covariance between two fuzzy numbers $\widetilde{A}$ and $\widetilde{B}$ is given by

$$
\begin{aligned}
\operatorname{Cov}_{f}(\widetilde{A}, \widetilde{B})=\frac{1}{2} \int_{0}^{1} f(\gamma) \\
\cdot\left[\left(\underline{a}(\gamma)-M_{f}(\widetilde{A})\right)\left(\underline{b}(\gamma)-M_{f}(\widetilde{B})\right)\right. \\
\left.+\left(\bar{a}(\gamma)-M_{f}(\widetilde{A})\right)\left(\widetilde{b}(\gamma)-M_{f}(\widetilde{B})\right)\right] d \gamma .
\end{aligned}
$$


Theorem 6. Let $\widetilde{A}$ and $\widetilde{B}$ be two fuzzy numbers and let $\lambda$, $\mu$ be positive numbers. Then the following conclusions can be obtained:

(i) $M_{f}(\lambda \widetilde{A} \pm \mu \widetilde{B})=\lambda M_{f}(\widetilde{A}) \pm \mu M_{f}(\widetilde{B})$;

(ii) $\operatorname{Var}_{f}(\lambda \widetilde{A} \pm \mu \widetilde{B})=\lambda^{2} \operatorname{Var}_{f}(\widetilde{A})+\mu^{2} \operatorname{Var}_{f}(\widetilde{B}) \pm$ $2 \lambda \mu \operatorname{Cov}_{f}(\widetilde{A}, \widetilde{B})$.

According to the definitions of the weighted possibilistic moments of fuzzy numbers, the sup-min extension principle, and Theorem 6, the following lemma can be obtained.

Lemma 7. Let $\widetilde{A}_{1}, \widetilde{A}_{2}, \ldots, \widetilde{A}_{n}$ be $n$ fuzzy numbers and let $\lambda_{1}, \lambda_{2}, \ldots, \lambda_{n}$ be $n$ real numbers. Then

(i) $M_{f}\left(\sum_{i=1}^{n} \lambda_{i} \widetilde{A}_{i}\right)=\sum_{i=1}^{n} \lambda_{i} M_{f}\left(\widetilde{A_{i}}\right)$;

(ii) $\operatorname{Var}_{f}\left(\sum_{i=1}^{n} \lambda_{i} \widetilde{A}_{i}\right) \quad=\quad \sum_{i=1}^{n} \lambda_{i}^{2} \operatorname{Var}_{f}\left(\widetilde{A}_{i}\right)+$ $2 \sum_{1 \leq i \neq j \leq n}^{n} \lambda_{i} \lambda_{j} \operatorname{Cov}_{f}\left(\widetilde{A}_{i}, \widetilde{A}_{j}\right)$.

Definition 8. Let $\widetilde{A}$ be a fuzzy number with weighted possibilistic mean $M_{f}(\widetilde{A})$. For any positive integer $r$, the $f$ weighted possibilistic moment ( $f$-WPM) of order $r$ about the possibilistic mean value of $\widetilde{A}$ is defined as $E_{r}(\widetilde{A})=$ $(1 / 2) \int_{0}^{1} f(r)\left[\left(a_{1}(\gamma)-M_{f}(\widetilde{A})\right)^{r}+\left(a_{2}(\gamma)-M_{f}(\widetilde{A})\right)^{r}\right] d \gamma$.

Definition 9. Let $\widetilde{A}$ be a fuzzy number, let $f(r)$ be a weighted function, and let $E_{r}(\widetilde{A})$ with $r=1,2, \ldots, n$ be weighted possibilistic moments of $\widetilde{A}$. Then the weighted possibilistic skewness (WPS) of fuzzy number $\widetilde{A}$ is defined as follows:

$$
\operatorname{Skewness}_{f}(\widetilde{A})=\operatorname{WPS}(\widetilde{A})=\frac{E_{3}(\widetilde{A})}{\left(\sqrt{E_{2}(\widetilde{A})}\right)^{3}} .
$$

The possibilistic skewness of a fuzzy number $\widetilde{A}$ shows the weight of fuzzy number at the left or right sides. Positive possibilistic skewness specifies that fuzzy number $\widetilde{A}$ is skewed to the right; that is, the tail on the right side is longer than the one on the left side. Negative possibilistic skewness specifies that fuzzy number $\widetilde{A}$ is skewed to the left; that is, the tail on the left side is longer than the one on the right side. A zero value indicates that fuzzy number $\widetilde{A}$ with the symmetric membership function, that is, its right tail and left tail, is equal.

In order to facilitate the calculation and reduce the computation complexity, we will use the third possibilistic moments as the possibilistic skewness of fuzzy number $\widetilde{A}$. We denoted it by Skewness $(\widetilde{A})=E_{3}(\widetilde{A})$. As well known, these two definitions of the possibilistic skewness about fuzzy number do not affect the way we study the problem.

According to the above definitions of weighted possibilistic moments, Theorem 6, and Lemma 7, we can get the following proposition.
Proposition 10. Let $\widetilde{r}(x)=\sum_{i=1}^{n} x_{i} \widetilde{r}_{i}=\left(\sum_{i=1}^{n} x_{i} c_{i}, \sum_{i=1}^{n} x_{i} d_{i}\right.$, $\left.\sum_{i=1}^{n} x_{i} \delta_{i}, \sum_{i=1}^{n} x_{i} \theta_{i}\right)$ be the portfolio return. The weighted possibilistic variance of $\widetilde{r}(x)$ is

$$
\begin{aligned}
\operatorname{Var}_{f}[\widetilde{r}(x)] \\
=\frac{\left[\sum_{i=1}^{n} x_{i}\left(\theta_{i}+\delta_{i}\right)\right]^{2}+\left[\sum_{i=1}^{n} x_{i}\left(\theta_{i}-\delta_{i}\right)\right]^{2}}{72} \\
+\left[\sum_{i=1}^{n} x_{i}\left(\frac{d_{i}-c_{i}}{2}+\frac{\theta_{i}+\delta_{i}}{6}\right)\right]^{2} .
\end{aligned}
$$

The possibilistic skewness of $\widetilde{r}(x)$ is

$$
\begin{aligned}
& \text { Ske }_{f}[\widetilde{r}(x)]=\frac{19}{1080}\left[\left(\sum_{i=1}^{n} x_{i} \theta_{i}\right)^{3}-\left(\sum_{i=1}^{n} x_{i} \delta_{i}\right)^{3}\right] \\
& +\frac{1}{24}\left[\sum_{i=1}^{n} x_{i}\left(d_{i}-c_{i}\right)\right]\left[\left(\sum_{i=1}^{n} x_{i} \theta_{i}\right)^{2}-\left(\sum_{i=1}^{n} x_{i} \delta_{i}\right)^{2}\right] \\
& +\frac{1}{72}\left[\left(\sum_{i=1}^{n} x_{i} \delta_{i}\right)\left(\sum_{i=1}^{n} x_{i} \theta_{i}\right)^{2}\right. \\
& \left.-\left(\sum_{i=1}^{n} x_{i} \theta_{i}\right)\left(\sum_{i=1}^{n} x_{i} \delta_{i}\right)^{2}\right] .
\end{aligned}
$$

2.3. Formulation Multiobjective Optimization Problems (MOPs). In this paper, the proposed portfolio selection problems will be modeled as multiobjective optimization problems (MOPs). Typically, a multiobjective optimization problem can be formulated as follows [27]:

$$
\begin{array}{cl}
\min & y=F(x)=\left[f_{1}(x), f_{2}(x), \ldots, f_{k}(x)\right] \\
\text { s.t. } & g_{j}(x) \geq 0, \quad j=1,2, \ldots, q \\
& h_{j}(x)=0, \quad j=q+1, \ldots, m .
\end{array}
$$

The feasible region $\mathbb{F}$ is defined as follows:

$$
\begin{aligned}
\mathbb{F} & =\left\{x \mid g_{j}(x) \geq 0, j=1,2, \ldots, q ; h_{j}(x)=0, j=q\right. \\
& +1, \ldots, m\} \subseteq S \subseteq R^{n} .
\end{aligned}
$$

Definition 11 (Pareto dominance). A solution $x^{*}=$ $\left(x_{1}^{*}, x_{2}^{*}, \ldots, x_{n}^{*}\right)$ is said to dominate (Pareto-optimal) another solution $x=\left(x_{1}, x_{2}, \ldots, x_{n}\right)$ (denoted by $\left.x^{*}>x\right)$, if both the conditions mentioned below are satisfied:

(i) $\forall j \in\{1,2, \ldots, k\}: f_{j}\left(x^{*}\right) \leq f_{j}(x)$;

(ii) $\exists j \in\{1,2, \ldots, k\}: f_{j}\left(x^{*}\right)<f_{j}(x)$.

Definition 12 (Pareto-optimal). A solution $x^{*} \in \mathbb{F}$ is said to be nondominated (Pareto-optimal)

if and only if $\neg \exists x$ such that $x>x^{*}$. 
Definition 13 (Pareto-optimal set). The set of all Paretooptimal solutions is defined as

$$
\mathrm{PS}=\left\{x^{*} \mid \neg \exists x \in \mathbb{F}: x>x^{*}\right\} .
$$

Definition 14 (Pareto-optimal front). The set of all Pareto solutions in objective space is defined as Pareto front and denoted by $\mathrm{PF}=\left\{\left[f_{1}(x), f_{2}(x), \ldots, f_{k}(x)\right] \mid x \in \mathrm{PS}\right\}$.

\section{Weighted Possibilistic Multiobjective Portfolio Selection Models}

In this section, we first introduce the problem description and notations will be used in the following section. Then, we will research the MVS and MVS-E multiobjective portfolio models.

3.1. Problem Description and Notations. Let us consider a multiobjective fuzzy portfolio selection problem with $n$ risk assets. The return rates and turnover rates of the risk assets are denoted as trapezoidal fuzzy numbers. For the notation convenience, we introduce the following notations:

$x_{i}$ : proportion of the total investment devoted to the risk asset $i, i=1, \ldots, n$;

$k_{i}$ : rate of transaction cost on the risk asset $i, i=1, \ldots, n$;

$\tilde{r}_{i}$ : fuzzy rate of return on the risk asset $i, i=1, \ldots, n$;

$\tilde{l}_{i}$ : fuzzy turnover rate of the risk asset $i, i=1,2, \ldots, n$.

In this paper, the transaction cost is assumed to be a $V$-shape function, which is the difference between a new portfolio $x=\left(x_{1}, x_{2}, \ldots, x_{n}\right)$ and a given portfolio $x^{0}=$ $\left(x_{1}^{0}, x_{2}^{0}, \ldots, x_{n}^{0}\right)$. For a new investor, it can be argued that $x_{i}^{0}=$ $0, i=1, \ldots, n$. Thus the total transaction cost of the portfolio is $\sum_{i=1}^{n} k_{i}\left|x_{i}-x_{i}^{0}\right|$.

For any risk asset, liquidity may be measured by using the turnover rate which is defined by the ratio of the average trading volume of the assets trade in the market and the trading volume of the tradable asset (i.e., shares held by the public) corresponding to the asset. It is well known that the future turnover rates of assets cannot be accurately predicted in the uncertain financial market. Therefore, the fuzzy set theory provides a new avenue to deal with this imprecision. The turnover rate of the $i$ th asset is regarded as a trapezoidal fuzzy number $\tilde{l}_{i}=\left(c_{i}, d_{i}, \delta_{i}, \theta_{i}\right), i=1,2, \ldots, n$ in this paper. Note that the turnover rate of the portfolio $x=$ $\left(x_{1}, x_{2}, \ldots, x_{n}\right)$ is $\widetilde{l}(x)=\sum_{i=1}^{n} \widetilde{l}_{i} x_{i}$. In our model, the portfolio liquidity is always greater than or equal to a tolerated level of fuzzy turnover rate $\widetilde{l}_{0}$ given by the investor; that is, $\widetilde{l}(x)=$ $\sum_{i=1}^{n} \widetilde{l}_{i} x_{i} \geq \tilde{l}_{0}$. According to the method for ranking fuzzy number reported in [28], the fuzzy inequation $\sum_{i=1}^{n} \widetilde{l}_{i} x_{i} \geq \widetilde{l}_{0}$ can be transformed into the crisp inequation $M\left(\sum_{i=1}^{n} \tilde{l}_{i} x_{i}\right) \geq$ $M\left(\widetilde{l}_{0}\right)$.

3.2. Construction of Fuzzy Multiobjective Portfolio Selection Model. For a rational investor, who wants to maximize both the expected return after paying transaction costs and skewness as well as minimizing the variance, the weighted possibilistic MVS three-objective model can be stated as

$$
\begin{array}{ll}
\max & f_{1}(x)=M_{f}\left[\sum_{i=1}^{n} \widetilde{r}_{i} x_{i}\right]-\sum_{i=1}^{n} k_{i}\left|x_{i}-x_{i}^{0}\right| \\
\min & f_{2}(x)=V_{f}\left[\sum_{i=1}^{n} \widetilde{r}_{i} x_{i}\right] \\
\max & f_{3}(x)=\operatorname{Ske}_{f}\left[\sum_{i=1}^{n} \widetilde{r}_{i} x_{i}\right] \\
& M\left(\sum_{i=1}^{n} \widetilde{l}_{i} \widetilde{x}_{i}\right) \geq M\left(\widetilde{l}_{0}\right) \\
\text { s.t. } & n \\
& \sum_{i=1}^{n} x_{i}=1, \quad x_{i} \geq 0, i=1,2, \ldots, n,
\end{array}
$$

where the first constraint means the liquidity of portfolio is greater than or equal to a given value $\widetilde{l}_{0}$ by the investor, and the second constraint implies that all the capital will be invested in $n$ assets and short-selling is not allowed.

It is not surprising that entropy is used as the divergence measure of asset portfolio in finance literature and its mathematical expression as follows:

$$
\operatorname{En}(x)=-\sum_{i=1}^{n} x_{i} \ln x_{i}
$$

where $x_{i}$ denotes the investment proportion of asset $i,(i=$ $1,2, \ldots, n)$. As can be seen the larger $\operatorname{En}(x)$ is, the more dispersed the investment is. When $x_{1}=x_{2}=\cdots=x_{n}=1 / n$, $\operatorname{En}(x)$ takes its maximum value.

Next, we add the proportion entropy to the weighted possibilistic MVS model for measuring the diversification degree. So, the well-diversified variation of the MVS model is denoted by the following weighted MVS-E four-objective model:

$$
\begin{array}{ll}
\max & f_{1}(x)=M_{f}\left[\sum_{i=1}^{n} \widetilde{r}_{i} x_{i}\right]-\sum_{i=1}^{n} k_{i}\left|x_{i}-x_{i}^{0}\right| \\
\min & f_{2}(x)=V_{f}\left[\sum_{i=1}^{n} \widetilde{r}_{i} x_{i}\right] \\
\max & f_{3}(x)=\operatorname{Ske}\left[\sum_{i=1}^{n} \widetilde{r}_{i} x_{i}\right] \\
\max & f_{4}(x)=-\sum_{i=1}^{n} x_{i} \ln x_{i} \\
\text { s.t. } & M\left(\sum_{i=1}^{n} \widetilde{l}_{i} \widetilde{x}_{i}\right) \geq M\left(\widetilde{l}_{0}\right) \\
& \sum_{i=1}^{n} x_{i}=1, \quad x_{i} \geq 0, i=1,2, \ldots, n .
\end{array}
$$


Corollary 15. Let $\widetilde{r}_{i}=\left(a_{i}, b_{i}, \alpha_{i}, \beta_{i}\right)$ and $\widetilde{l}_{i}=\left(c_{i}, d_{i}, \delta_{i}, \theta_{i}\right)$ represent the fuzzy returns and the fuzzy turnover rates of the ith asset for $i=1,2, \ldots, n$, and $\widetilde{l}_{0}=\left(c_{0}, d_{0}, \delta_{0}, \theta_{0}\right)$ is a given fuzzy turnover rate confidence level by the investor. Then the MVS model and MVS-E model can be converted into the following deterministic MOPs, respectively:

$$
\begin{aligned}
& \max f_{1}(x)=\left[\sum_{i=1}^{n}\left(\frac{a_{i}+b_{i}}{2}+\frac{\beta_{i}-\alpha_{i}}{6}\right) x_{i}-\sum_{j=1}^{n} k_{i}\left|x_{i}-x_{i}^{0}\right|\right] \\
& \min f_{2}(x) \\
& =\frac{\left[\sum_{i=1}^{n} x_{i}\left(\beta_{i}+\alpha_{i}\right)\right]^{2}+\left[\sum_{i=1}^{n} x_{i}\left(\beta_{i}-\alpha_{i}\right)\right]^{2}}{72} \\
& +\left[\sum_{i=1}^{n} x_{i}\left(\frac{b_{i}-a_{i}}{2}+\frac{\beta_{i}+\alpha_{i}}{6}\right)\right]^{2} \\
& =\frac{19}{1080}\left[\left(\sum_{i=1}^{n} x_{i} \beta_{i}\right)^{3}-\left(\sum_{i=1}^{n} x_{i} \alpha_{i}\right)^{3}\right] \\
& +\frac{1}{24}\left[\sum_{i=1}^{n} x_{i}\left(b-a_{i}\right)\right]\left[\left(\sum_{i=1}^{n} x_{i} \beta_{i}\right)^{2}-\left(\sum_{i=1}^{n} x_{i} \alpha_{i}\right)^{2}\right] \\
& +\frac{1}{72}\left[\left(\sum_{i=1}^{n} x_{i} \alpha_{i}\right)\left(\sum_{i=1}^{n} x_{i} \beta_{i}\right)^{2}-\left(\sum_{i=1}^{n} x_{i} \beta_{i}\right)\left(\sum_{i=1}^{n} x_{i} \alpha_{i}\right)^{2}\right] \\
& \text { s.t. } \quad \sum_{i=1}^{n}\left(\frac{c_{i}+d_{i}}{2}+\frac{\theta_{i}-\delta_{i}}{6}\right) x_{i}-\left(\frac{c_{0}+d_{0}}{2}+\frac{\theta_{0}-\delta_{0}}{6}\right) \geq 0 \\
& \sum_{i=1}^{n} x_{i}=1, \quad x_{i} \geq 0, i=1,2, \ldots, n, \\
& \max f_{1}(x)=\left[\sum_{i=1}^{n}\left(\frac{a_{i}+b_{i}}{2}+\frac{\beta_{i}-\alpha_{i}}{6}\right) x_{i}-\sum_{j=1}^{n} k_{i}\left|x_{i}-x_{i}^{0}\right|\right] \\
& \min f_{2}(x) \\
& =\frac{\left[\sum_{i=1}^{n} x_{i}\left(\beta_{i}+\alpha_{i}\right)\right]^{2}+\left[\sum_{i=1}^{n} x_{i}\left(\beta_{i}-\alpha_{i}\right)\right]^{2}}{72} \\
& +\left[\sum_{i=1}^{n} x_{i}\left(\frac{b_{i}-a_{i}}{2}+\frac{\beta_{i}+\alpha_{i}}{6}\right)\right]^{2}
\end{aligned}
$$

$\max$

$$
\begin{aligned}
&= \frac{19}{1080}\left[\left(\sum_{i=1}^{n} x_{i} \beta_{i}\right)^{3}-\left(\sum_{i=1}^{n} x_{i} \alpha_{i}\right)^{3}\right] \\
&+\frac{1}{24}\left[\sum_{i=1}^{n} x_{i}\left(b_{i}-a_{i}\right)\right]\left[\left(\sum_{i=1}^{n} x_{i} \beta_{i}\right)^{2}-\left(\sum_{i=1}^{n} x_{i} \alpha_{i}\right)^{2}\right] \\
&+\frac{1}{72}\left[\left(\sum_{i=1}^{n} x_{i} \alpha_{i}\right)\left(\sum_{i=1}^{n} x_{i} \beta_{i}\right)^{2}-\left(\sum_{i=1}^{n} x_{i} \beta_{i}\right)\left(\sum_{i=1}^{n} x_{i} \alpha_{i}\right)^{2}\right] \\
& \max \quad f_{4}(x)=-\sum_{i=1}^{n} x_{i} \ln x_{i} \\
& \text { s.t. } \quad \sum_{i=1}^{n}\left(\frac{c_{i}+d_{i}}{2}+\frac{\theta_{i}-\delta_{i}}{6}\right) x_{i}-\left(\frac{c_{0}+d_{0}}{2}+\frac{\theta_{0}-\delta_{0}}{6}\right) \geq 0 \\
& \quad \sum_{i=1}^{n} x_{i}=1, \quad x_{i} \geq 0, i=1,2, \ldots, n .
\end{aligned}
$$

Proof. $\widetilde{r}_{i}=\left(a_{i}, b_{i}, \alpha_{i}, \beta_{i}\right)$ and $\widetilde{l}_{i}=\left(c_{i}, d_{i}, \delta_{i}, \theta_{i}\right)$ are trapezoidal fuzzy number for $i=1,2, \ldots, n$.

According to the extension principle of Zadeh, $\sum_{i=1}^{n} \widetilde{r}_{i} x_{i}=\left(\sum_{i=1}^{n} x_{i} a_{i}, \sum_{i=1}^{n} x_{i} b_{i}, \sum_{i=1}^{n} x_{i} \alpha_{i}, \sum_{i=1}^{n} x_{i} \beta_{i}\right)$ and $\sum_{i=1}^{n} \tilde{l}_{i} x_{i}=\left(\sum_{i=1}^{n} x_{i} c_{i}, \sum_{i=1}^{n} x_{i} d_{i}, \sum_{i=1}^{n} x_{i} \delta_{i}, \sum_{i=1}^{n} x_{i} \theta_{i}\right)$ are also trapezoidal fuzzy numbers for $i=1,2, \ldots, n$. Combining this with Lemma 7 and Proposition 10, we can draw this corollary.

\section{A New Multiobjective Evolutionary Algorithm for Portfolio Selection}

The diversity of solutions is an important aspect for multiobjective evolutionary algorithms and portfolio selection problems. To well maintain the diversity, the solution location (or distribution) of the obtained nondominated solution set in the objective space is considered when the algorithm makes the solutions replacement. Based on the idea, a multiobjective evolutionary algorithm is designed to solve the portfolio selection problems. In this section, we will introduce the proposed new algorithm which includes three parts: solutions classification, update strategy, and selection strategy.

4.1. Solutions Classification. By using a set of direction vectors, the objective space of MPOs is decomposed into a set of subobjective spaces, and obtained solutions are classified, so that there is surely a solution in each subobjective space. For a given set of direction vectors $\left(\lambda^{1}, \lambda^{2}, \ldots, \lambda^{N}\right)$ and the set of current obtained solutions being POP, these solutions will be classified by the following formula:

$$
\begin{aligned}
P^{i} & =\left\{x \mid x \in \mathrm{POP}, \Delta\left(F(x), \lambda^{i}\right)\right. \\
& \left.=\max _{1 \leq j \leq N}\left\{\Delta\left(F(x), \lambda^{j}\right)\right\}\right\}, \\
\Delta & \left(F(x), \lambda^{i}\right)=\frac{\lambda^{i} *(F(x)-Y)^{T}}{\left\|\lambda^{i}\right\| *\|F(x)-Y\|}, \quad i=1, \ldots, m .
\end{aligned}
$$

In the above formula, $Y=\left(Y_{1}, \ldots, Y_{m}\right)$ is a reference point and $Y_{i}=\min \left\{f_{i}(x) \mid x \in \mathbb{F}\right\}, \Delta\left(F(x), \lambda^{i}\right)$ is the cosine of the angle between $\lambda^{i}$ and $F(x)-Y$. These solutions are separated into $N$ classes by formula (14) and the objective space $\Omega$ is separated into $N$ subobjective spaces $\Omega_{1}, \ldots, \Omega_{N}$, and $\Omega_{k}(k=1, \ldots, N)$ is

$$
\begin{aligned}
\Omega_{k} & =\left\{F(x) \mid x \in \mathbb{F}, \Delta\left(F(x), \lambda^{k}\right)\right. \\
& \left.=\max _{1 \leq j \leq N}\left\{\Delta\left(F(x), \lambda^{j}\right)\right\}\right\} .
\end{aligned}
$$

When $P^{i}(1 \leq i \leq N)$ is empty, a solution is randomly picked from POP and placed into $P^{i}$. The following comments are given on the classification (decomposition) method. 
The method is equivalent in a way that the PFs of all these subobjective spaces constitute the PFs of the deterministic MOPs (Corollary 15).

Even if the PSs of the deterministic MOPs have nonlinear geometric shape, the PS of each subobjective space could be approaching linear geometric shape, because it is just a small part of the PSs of problems (12) and (13). Thus, formulas (14) and (15) make problems (12) and (13) simpler than before, at least in accordance with PS shapes.

This classification (decomposition) method does not need any aggregation method. A user only needs to select a set of direction vectors. To some degree, it needs little human labor.

4.2. Update Strategy. We use the elitist strategy to update solutions. If it satisfies one of the following conditions, a new solution will take the place of the current solution to a subobjective space.

If the objective vector of the current solution is not located in this subjective space, the objective vector of the new solution is located in this space or the current solution is dominated by the new solution.

If the objective vector of the current solution lies in this subjective space, the objective vector of the new solution also lies in this space and the current solution is dominated by the new solution.

The first update condition makes sure that there is a solution in each subobjective space where the solution's objective vector is located, which can maintain the diversity of obtained solutions well. The second update condition makes sure that nondominated solution is retained, which lets solutions converge to the PF.

4.3. Selection Strategy. In this section, we hope that those solutions which are retained after the above update strategy and dominated by other solutions have stronger survival ability than other solutions. In this way, these solutions are more possibly selected to generate new solutions, and then their subobjective spaces are able to quickly seek out their optimal solutions. Therefore, the finally obtained solution to each subobjective space is as close as possible to the PF. To reach the goal, by using the crowding distance [20], the fitness value of a solution is computed for the selection operators. Because these solutions are dominated by other solutions and the objective vectors of those solutions do not lie in these subobjective spaces of these solutions, according to the objective vector, these solutions have fewer solutions in their surroundings than other solutions. Thus, by using the crowding distance to compute the fitness value of a solution, the fitness values of these solutions are better than those of other solutions and these solutions are more possible to be chosen to generate new solutions.

4.4. Steps of the Proposed Algorithm. According to the above methods, a new multiobjective evolutionary algorithm is proposed and the steps of the algorithm are followed.

Step 1 (initialization). Given that $N$ direction vectors $\left(\lambda^{1}, \lambda^{2}, \ldots, \lambda^{N}\right)$ randomly produce an initial population
$\operatorname{POP}(k)$ and its size is $N$, let $k=0$. Set $Y_{i}=\min \left\{f_{i}(x) \mid x \in\right.$ $\operatorname{POP}(k)\}, \quad 1 \leq i \leq m$.

Step 2 (fitness). Solutions of $\operatorname{POP}(k)$ are firstly separated into $N$ classes by formula (14) and the fitness value of each solution in $\operatorname{POP}(k)$ is computed by the crowding distance. Then some better solutions are picked from the population $\operatorname{POP}(k)$ and put into the population POP. In this section, binary tournament selection is used.

Step 3 (new solutions). Use genetic operators to the parent population to generate offspring. The set of all these offspring is expressed as $O$.

Step 4 (update). $Y$ is firstly updated. For each $j=1, \ldots, m$, if $Y_{j}>\min \left\{f_{j}(x) \mid x \in O\right\}$, then set $Y_{j}=\min \left\{f_{j}(x) \mid x \in O\right\}$. The solutions of $\operatorname{POP}(k) \cup O$ are firstly classed by formula (14); then $N$ best solutions are chosen by the update strategy of Section 4.2 and put into $\operatorname{POP}(k+1)$. Let $k=k+1$.

Step 5 (termination). If stopping criteria are met, then stop; otherwise, go to Step 2.

The major differences among the proposed algorithm, NSGA-II, and MOEA/D include the following: MOEA/D and NSGA-II make their selection by aggregation functions and nondominance ranking. Therefore, some subproblems may receive no solutions for the next generation, as it is very likely to happen, which is because obtained solutions of these subregions are completely dominated by some solutions of obtained nondominated solutions. As a result, NSGA-II and MOEA/D may lose the diversity of population. However, each subproblem of the proposed algorithm has a feasible objective region and feasible solutions are better than infeasible solutions. The proposed algorithm considers the location (and distribution) of solutions in the solution set in the objective space to well maintain the diversity.

\section{Numerical Examples and Analysis}

In this section, in order to demonstrate the effectiveness of the modeling idea and the proposed algorithm, we first compare our algorithm with two famous algorithms: nondominated sorting genetic algorithm II (NSGA-II [20]) and multiobjective evolutionary algorithm based on decomposition (MOEA/D [21]) through some numerical examples based on the data of the Shanghai Stock Exchange Market. Then, we use our proposed algorithm to solve the weighted possibilistic portfolio selection models MVS and MVS-E. Finally, the result analysis will be given between the MVS model and MVS-E model by these numerical examples.

5.1. Data Processing. We assume that an investor chooses 12 assets from Shanghai Stock Exchange for his or her investment. The exchange codes of the 12 assets are 601098, 601880, 600563, 600038, 601888, 601377, 600721, 600681, $600571,600419,600570$, and 600201. For convenience of description, we denote the 12 assets successively as Assets 1, 2, $3,4,5,6,7,8,9,10,11$, and 12 in the example. Here, we collect 
TABLE 1: Sample statistics for the weekly returns on the assets (20122015) from historical data.

\begin{tabular}{lcccc}
\hline Returns & $\begin{array}{c}\text { 5th } \\
\text { percentile }\end{array}$ & $\begin{array}{c}\text { 40th } \\
\text { percentile }\end{array}$ & $\begin{array}{c}\text { 60th } \\
\text { percentile }\end{array}$ & $\begin{array}{c}\text { 95th } \\
\text { percentile }\end{array}$ \\
\hline Asset 1 & -0.0627 & -0.0065 & 0.0188 & 0.0869 \\
Asset 2 & -0.0626 & -0.0094 & 0.0077 & 0.1111 \\
Asset 3 & -0.0798 & -0.0062 & 0.0219 & 0.0913 \\
Asset 4 & -0.0709 & -0.0076 & 0.0154 & 0.0951 \\
Asset 5 & -0.0609 & -0.0047 & 0.0144 & 0.0804 \\
Asset 6 & -0.0804 & -0.0093 & 0.0189 & 0.1143 \\
Asset 7 & -0.0867 & -0.0053 & 0.0180 & 0.0975 \\
Asset 8 & -0.0861 & -0.0044 & 0.0228 & 0.0992 \\
Asset 9 & -0.0859 & -0.0029 & 0.0271 & 0.1177 \\
Asset 10 & -0.0868 & -0.0124 & 0.0199 & 0.1457 \\
Asset 11 & -0.0700 & 0.0063 & 0.0139 & 0.1215 \\
Asset 12 & -0.0571 & -0.0060 & 0.01930 & 0.0939 \\
\hline
\end{tabular}

original data from the weekly closing pricing and turnover rate of these assets in three years from January 2012 to January 2015 as the sample data. Using the simple estimation method in Vercher et al. [29], we get the statistics of the historical data of 12 rates. Table 1 provides the summary statistics of the data.

In our numerical example, we assume that the transaction costs of assets are identical, that is, $k_{i}=0.003$, for all $i=$ $1,2, \ldots, 12$, and the fuzzy turnover rate confidence level given by the investor is $\widetilde{l}_{0}=(0.0227,0.0322,0.0658,0.2324)$.

For the fuzzy return rates of the models, we use the sample percentiles to approximate the core and spreads of the trapezoidal fuzzy returns on the assets. In fact, we have decided to set the core $\left[a_{i}, b_{i}\right]$ of the fuzzy return $\widetilde{r}_{i}$ as the interval [40th, 60th] and the quantities 40th-5th and 95th-60th as the left $\alpha_{i}$ and right $\beta_{i}$ spreads, respectively, where $k$ th is the $k$ th percentile of the sample. Then, the corresponding membership function is given by Definition 1 . Consider

$$
\mu_{\widetilde{r}_{i}}(x)= \begin{cases}1-\frac{a_{i}-x}{\alpha_{i}}, & \text { if } a_{i}-\alpha_{i} \leq x \leq a_{i} \\ 1, & \text { if } a_{i} \leq x \leq b_{i} \\ 1-\frac{x-d}{\beta_{i}}, & \text { if } b_{i} \leq x \leq b_{i}+\beta_{i} \\ 0, & \text { if otherwise. }\end{cases}
$$

In the same way, we deal with the fuzzy turnover rates of $i_{-}$th asset by using the historical data. Then, the parameters of the fuzzy return rate $\widetilde{r}_{i}=\left(a_{i}, b_{i}, \alpha_{i}, \beta_{i}\right)$ and the turnover rates $\tilde{l}_{i}=\left(c_{i}, d_{i}, \delta_{i}, \theta_{i}\right)$ of the $i$ th asset are shown, respectively, in Tables 2 and 3 .

5.2. Parameter Settings. The models are executed on a Genuine Intel CPU W35052.53 GHz and a 4.0 GB RAM personal computer with MATLAB software. The individuals are all coded as the real vectors. Polynomial mutation [30] operators are applied directly to real parameter values in three algorithms, that is, our algorithm, NSGA-II, and MOEA/D. For crossover operators, simulated binary crossover (SBX [30]) is used in NSGA-II and our algorithm; differential evolution (DE) [31] is used in MOEA/D. The parameter settings in this paper are as follows.

(1) Control parameters in reproduction operators:

(a) Distribution index is 20 and crossover probability is 1 in the SBX operator.

(b) Crossover rate is 1.0 and scaling factor is 0.5 in the DE operator.

(c) Distribution index is 20 and mutation probability is 0.1 in mutation operator.

(2) The population size is 105 in the three algorithms. Weight vectors are generated by using the method which is used in [21]. The direction vectors of our algorithm are the weight vectors. The number of weight vectors is the same as the population size. The number of the weight vectors in the neighborhood in MOEA/D is 20 for all test problems. The Tchebycheff approach [21] is used for MOEA/D as an aggregate function. Other control parameters in MOEA/D are the same as those in [21].

(3) Each algorithm is run 30 times independently for each model. All the three algorithms stop after 1000 generations.

5.3. Comparisons of Our Algorithm with MOEA/D and NSGAII. In this subsection, the comparisons of our algorithm with MOEA/D and NSGA-II algorithms will be given through some numerical examples based on the data of the Shanghai Stock Exchange Market. Additionally, different parameters are considered in two case studies: (a) returns of all assets become more increasing up to 10 percent than the sample returns in Table 2; (b) returns of all assets become more decreasing up to 10 percent than the sample returns in Table 2.

In order to compare the performance of the different algorithms quantitatively, performance metrics are needed. In this paper, the following performance metrics are used to compare the performance of the different algorithms quantitatively: set coverage ( $C$-metric) [32] and hypervolume indicator (HV) [33]. C-metric is defined as the percentage of the solutions in $B$ that are dominated by at least one solution in $A$; that is, $C(A, B)=\mid\{u \in B \mid \exists v \in A$ : $v$ dominates $u\}|/| B \mid ; C(A, B)$ is not necessarily equal to $1-$ $C(A, B) \cdot C(A, B)=1$ means that all solutions in $B$ are dominated by some solutions in $A$, while $C(A, B)=0$ implies that no solution in $B$ is dominated by a solution in $A$. The hypervolume indicator has been used widely in evolutionary multiobjective optimization to evaluate the performance of search algorithms. It computes the volume of the dominated portion of the objective space relative to a reference point. Higher values of this performance indicator imply more desirable solutions. The hypervolume indicator measures both the convergence to the true Pareto front and diversity of the obtained solutions.

To visually compare the performance of the three algorithms, the obtained nondominated solutions by them on 
TABLE 2: Fuzzy returns of 12 assets.

\begin{tabular}{lcccc}
\hline Asset $i$ & 1 & 2 & 3 & 4 \\
\hline \multirow{2}{*}{ Return rate } & $(-0.0065,0.0188$, & $(-0.0094,0.0077$, & $(-0.0062,0.0219$, & $(-0.0076,0.0154$, \\
& $0.0562,0.0681)$ & $0.0532,0.1034)$ & $0.0736,0.0694)$ & 7 \\
\hline Asset $i$ & 5 & 6 & $(-0.0053,0.0180$, & $0.0633,0.0797)$ \\
\hline \multirow{2}{*}{ Return rate } & $(-0.0047,0.0144$, & $(-0.0093,0.0189$, & $(-0.0044,0.0228$, \\
& $0.0562,0.0660)$ & $0.0711,0.0954)$ & $0.0814,0.0795)$ & $0.0817,0.0764)$ \\
\hline Asset $i$ & 9 & 10 & 11 & 12 \\
\hline \multirow{2}{*}{ Return rate } & $(-0.0029,0.0271$, & $(-0.0124,0.0199$, & $(0.0063,0.0139$, & $(-0.0060,0.0193$, \\
& $0.0830,0.0906)$ & $0.0744,0.1258)$ & $0.0637,0.1076)$ & $0.0511,0.0746)$ \\
\hline
\end{tabular}

TABLE 3: Fuzzy turnover rates of 12 assets.

\begin{tabular}{lcccc}
\hline Stock $i$ & 1 & 2 & 3 & 4 \\
Turnover rate & $(0.0416,0.0662$, & $(0.0434,0.0639$, & $(0.0526,0.0657$, & $(0.0508,0.0723$, \\
& $0.0224,0.01315)$ & $0.0352,0.2148)$ & $0.0290,0.0599)$ & 7 \\
\hline Asset $i$ & 5 & 6 & $(0.0723,0.0990$, & $0.0338,0.0994)$ \\
\hline \multirow{2}{*}{ Turnover rate } & $(0.0220,0.0278$, & $(0.0449,0.0699$, & $(0.0708,0.0954$, \\
& $0.0124,0.0571)$ & $0.0239,0.1900)$ & $0.0481,0.1264)$ & $0.0426,0.1297)$ \\
\hline Asset $i$ & 9 & 10 & 11 & 12 \\
\hline \multirow{2}{*}{ Turnover rate } & $(0.0499,0.0820$, & $(0.0705,0.0970$, & $(0.0299,0.0503$, & $(0.0290,0.0379$, \\
& $0.0315,0.0965)$ & $0.0515,0.2344)$ & $0.0194,0.0875)$ & $0.0164,0.0590)$ \\
\hline
\end{tabular}

MVS model are shown in Figure 1. Figure 2 shows the comparison of the nondominated solutions obtained by our proposed algorithm with MOEA/D and NSGA-II. Figure 2(a) illustrates the comparison of Pareto fronts of our proposed algorithm with NSGA-II, and Figure 2(b) shows the comparison of Pareto fronts of our proposed algorithm with MOEA/D.

Obviously, both NSGA-II and MOEA/D cannot locate the diversity and uniformity nondominated solutions compared with our algorithm. In contrast, our algorithm can obtain a diverse population of nondominated solutions. These figures indicate that, in both the aspects of uniformity and distribution of solutions, our algorithm gives a better performance than the NSGA-II and MOEA/D.

Table 4 presents the means of $\mathrm{C}$-metric value finally obtained by our algorithm, MOEA/D, and NSGA-II for the two models. It clearly shows that all of the solutions obtained by our algorithm dominate the solutions obtained by the NSGA-II and MOEA/D.

Table 5 presents the mean and standard deviation of $\mathrm{HV}$ obtained by our algorithm, MOEA/D, and NSGA-II. Best solutions obtained are written in bold in this table. In terms of the mean value of $\mathrm{HV}$, the values of $\mathrm{HV}$ obtained by our algorithm are much bigger than those obtained by the other two algorithms, which illustrates that the diversities of solutions obtained by our algorithm are better than those obtained by MOEA/D and NSG-AII.

5.4. Portfolio Performance Evaluation. Performance measures are important tools for management decisions. As a traditional performance measure, the Sharpe ratio (SR) is
TABLE 4: Average set coverage between our algorithm (A), MOEA/D $(B)$, and NSGA-II $(E)$.

\begin{tabular}{lcccc}
\hline$C$-metric & $C(A, B)$ & $C(B, A)$ & $C(A, E)$ & $C(E, A)$ \\
\hline MVS model & 0.7333 & 0 & 0.7552 & 0 \\
MVS-E model & 0.7247 & 0 & 0.9689 & 0 \\
\hline Case (a) & & & & \\
$\quad$ MVS model & 0.8181 & 0 & 0.3298 & 0.0051 \\
$\quad$ MVS-E model & 0.7308 & 0 & 0.8950 & 0 \\
\hline Case (b) & & & & \\
$\quad$ MVS model & 0.9514 & 0 & 0.1533 & 0 \\
$\quad$ MVS-E model & 0.8085 & 0 & 0.9885 & 0 \\
\hline
\end{tabular}

a commonly used measure of portfolio performance and its formula is given as the following general form:

$$
\mathrm{SR}=\frac{E[r(x)]}{\sqrt{V(r(x))}},
$$

where $r(x)$ is the return of portfolio. However, since the SR is based on the mean-variance theory, it is valid only for either normally distributed returns or quadratic preferences. Particularly, the SR can lead to misleading conclusions when the return distributions are skewed or present heavy tails [34]. Zakamouline and Koekebakker [35] proposed that the adjusted skewness Sharpe ratio (ASR) is a prominent performance measure for measuring portfolio performance in the mean-variance-skewness framework of portfolio model. The adjusted skewness Sharpe ratio (ASR), which takes into account the skewness of portfolio, is defined as follows:

$$
\mathrm{ASR}=\mathrm{SR} \sqrt{1+\frac{\operatorname{Ske}[r(x)]}{3} \mathrm{SR}},
$$






(a)

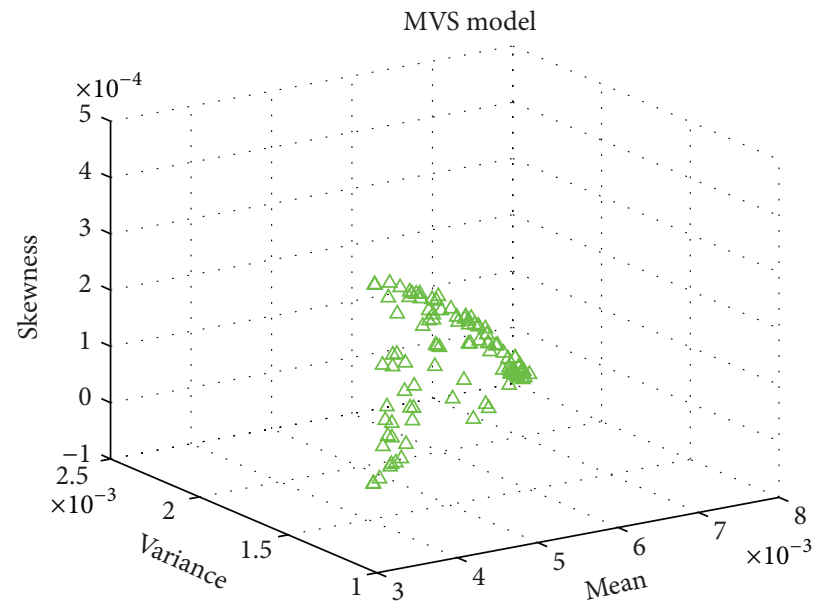

(b)

MVE model

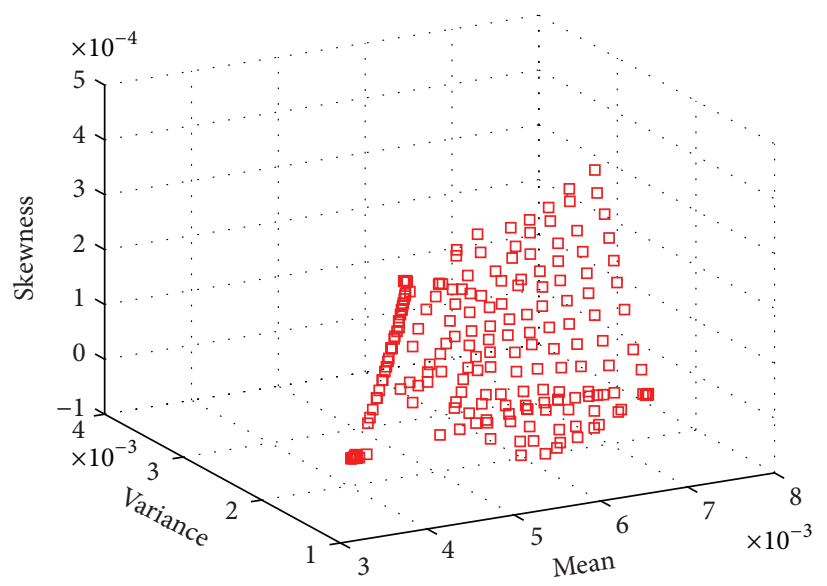

(c)

Figure 1: (a) NSGA-II, (b) MOEA/D, and (c) our algorithm.

TABLE 5: HV obtained by our algorithm, MOEA/D, and NSGA-II.

\begin{tabular}{|c|c|c|c|c|}
\hline \multirow{2}{*}{$\mathrm{HV}$} & \multicolumn{2}{|c|}{ MVS model } & \multicolumn{2}{|c|}{ MVS-E model } \\
\hline & Mean & SD & Mean & $\mathrm{SD}$ \\
\hline Our algorithm & $0.2258 \mathrm{e}-005$ & $0.0003 e-006$ & $0.1184 e-004$ & $0.0091 e-006$ \\
\hline MOEA/D & $0.1697 e-005$ & $0.0255 e-006$ & $0.0669 e-004$ & $0.3620 e-006$ \\
\hline NSGA-II & $0.1448 e-005$ & $0.147 e-006$ & $0.0292 e-004$ & $0.2114 e-006$ \\
\hline \multicolumn{5}{|l|}{ Case (a) } \\
\hline Our algorithm & $0.0167 e-004$ & $0.0321 e-008$ & $0.1175 e-004$ & $0.9596 e-008$ \\
\hline MOEA/D & $0.5385 e-005$ & $0.1454 e-005$ & $0.5726 e-005$ & $0.0391 e-005$ \\
\hline NSGA-II & $0.1453 e-005$ & $0.1338 e-006$ & $0.2924 e-005$ & $0.1746 e-006$ \\
\hline \multicolumn{5}{|l|}{ Case (b) } \\
\hline Our algorithm & $0.0166 e-004$ & $0.0044 e-007$ & $0.1184 e-004$ & $0.1537 e-007$ \\
\hline MOEA/D & $0.5882 e-005$ & $0.1656 e-005$ & $0.7524 e-005$ & $0.0293 e-005$ \\
\hline NSGA-II & $0.1386 e-005$ & $0.1405 e-006$ & $0.2941 e-005$ & $0.2103 e-006$ \\
\hline
\end{tabular}




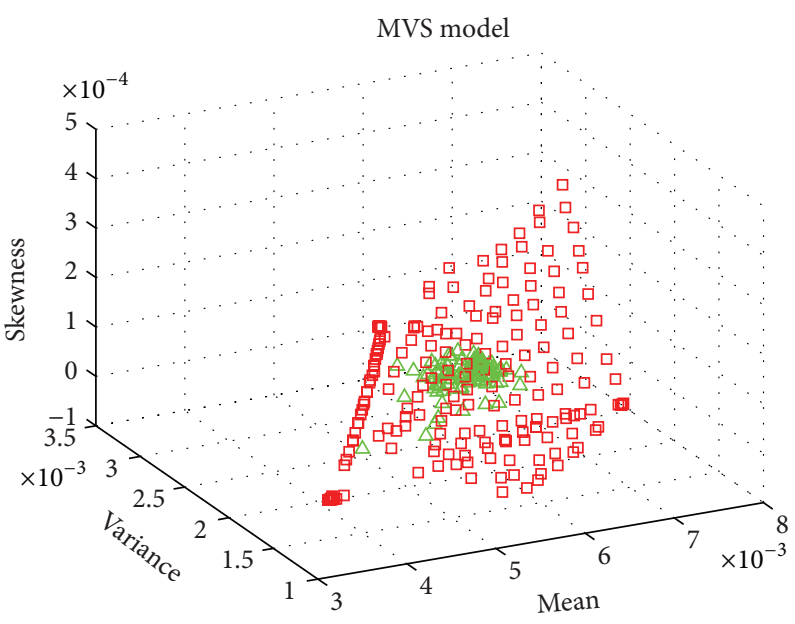

$\triangle$ NSGA-II

$\square$ Our algorithm

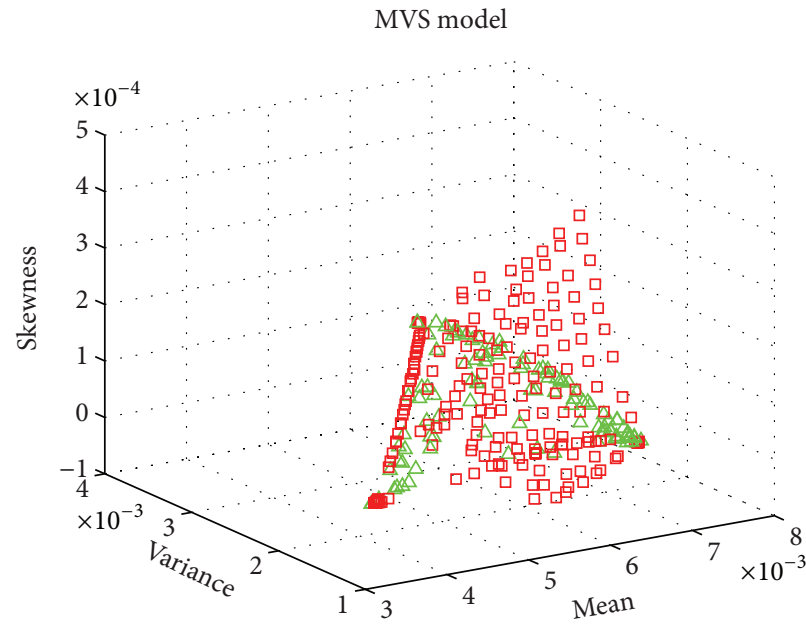

$\triangle$ MOEA/D

口 Our algorithm

FIGURE 2: (a) Solutions obtained by NSGA-II and our algorithm; (b) solutions obtained by MOEA/D and our algorithm.

TABLE 6: Summary statistics of ASR for MVS and MVS-E model.

\begin{tabular}{lccccc}
\hline ASR & Min & Max & Mean & Median & SD \\
\hline MVS model & 0.0653 & 0.1617 & 0.1186 & 0.1168 & 0.0250 \\
MVS-E model & 0.0958 & 0.1619 & 0.1313 & 0.1334 & 0.0195 \\
\hline
\end{tabular}

where Ske $[r(x)]$ is the skewness of portfolio. Now we observe that the value of ASR can reflect the level of maximum expected utility. Therefore, it can also be used as a performance measure. The larger the value of ASR is, the better the investment portfolio is, and the better the performance of the portfolio model is. In this paper, we consider the optimal investment under the criterion of mean-variance-skewness framework. So we use the ASR to evaluate the performance of our portfolio models.

We use our new algorithm to solve the models MVS and MVS-E, respectively. Table 6 presents the statistics of ASR for each model, including min, max, mean, median, and standard deviation of the ASR. We can see that the various statistical indicators of ASR of MVS-E are larger than the corresponding MVSs. It means that the addition of entropy function increases the efficiency of mean-variance-skewness model; that is, the MVS-E model outperforms the MVS model in terms of ASR performance measure.

Figure 3 shows boxplots of the ASRs indicator for the MVS and MVS-E model; in Figure 3, the symbol " $\triangle$ " represents the mean of ASR. The box in the plot contains $50 \%$ of the data points from the 25th to 75 th percentile, and the red line drawn across the box is the median of ASR. The whiskers are lines extending above and below each box. It should be noted that MVS-E exhibits notably better results than the MVS model from Figure 3. Taking into account the result of Table 6 and Figure 3 together, it means that the MVS-E



FIgURE 3: Boxplot of the ASR indicator for MVS and MVS-E.

model can enhance diversity of the portfolios and make asset allocation more feasible than the traditional MVS model.

Figure 4 shows the boxplots of average portfolio allocations that correspond to the portfolio models (MVS and MVS-E) in the last generation, where $x_{i}$ is the percentage of the sharing fund corresponding to the $i$ th asset, and others. In Figure 4, the symbol " $\triangle$ " represents the mean of $x_{i}$, the box in the plot contains $50 \%$ of the data points from the 25th to 75th percentile, and the red line drawn across the box is the median of $x_{i}$. The whiskers are lines extending above and below each box. Figure 4(a) shows that for the MVS model the Pareto-optimal solutions allocate an average $43.67 \%$ of investment weights to the 2 th asset $\left(x_{2}\right)$, allocate an average $19.42 \%$ of investment weights to the 5 th asset $\left(x_{5}\right)$, and allocate an average $36.45 \%$ of investment weights to the 9th asset $\left(x_{9}\right)$.

As can be seen from Figure 4(a), about $99.54 \%$ of investment weights concentrated on the three assets, and the optimal investment allocation solution in the MVS model 
TABLE 7: The different patterns of investment of MVS model.

\begin{tabular}{lcccccccccccccccc}
\hline Investing & 1 & 2 & 3 & 4 & 5 & 6 & 7 & 8 & 9 & 10 & 11 & 12 & Return & Risk & Skewness \\
\hline$P 1$ & 0 & 0.0100 & 0 & 0 & 0 & 0 & 0 & 0 & 0.9900 & 0 & 0 & 0 & 0.075 & 0.0023 & 0.0004 \\
$P 2$ & 0 & 0.0359 & 0 & 0 & 0 & 0 & 0 & 0 & 0.9641 & 0 & 0 & 0 & 0.074 & 0.0022 & 0.0003 \\
\hline$P 3$ & 0 & 0.6885 & 0 & 0 & 0 & 0 & 0 & 0 & 0.3115 & 0 & 0 & 0 & 0.0052 & 0.0016 & 0.0002 \\
$P 4$ & 0 & 0.6887 & 0 & 0 & 0 & 0 & 0 & 0 & 0.3112 & 0 & 0 & 0 & 0.0050 & 0.0015 & 0.0001 \\
\hline$P 5$ & 0 & 0.0301 & 0 & 0 & 0.9672 & 0 & 0 & 0 & 0 & 0 & 0 & 0 & 0.0034 & 0.0012 & 0 \\
$P 6$ & 0 & 0.0284 & 0 & 0 & 0.9717 & 0 & 0 & 0 & 0 & 0 & 0 & 0 & 0.0032 & 0.0011 & 0 \\
\hline
\end{tabular}

TABLE 8: IGD, GD, and HV obtained by the proposed algorithm, MOEA/D, NSGA-II, and D2MOPSO.

\begin{tabular}{|c|c|c|c|c|c|c|c|c|}
\hline \multirow{2}{*}{ Instance } & \multicolumn{2}{|c|}{ The proposed algorithm } & \multicolumn{2}{|c|}{ MOEA/D } & \multicolumn{2}{|c|}{ NSGA-II } & \multicolumn{2}{|c|}{ D2MOPSO } \\
\hline & Mean & Std. & Mean & Std. & Mean & Std. & Mean & Std. \\
\hline \multicolumn{9}{|l|}{ ZDT1 } \\
\hline IGD & 0.0040 & 0.0002 & $0.0040(=)$ & 0.0003 & $0.0042(=)$ & 0.0003 & $0.0041(=)$ & 0.0001 \\
\hline GD & 0.0009 & 0.0001 & $0.0010(=)$ & 0.0002 & $0.0010(=)$ & 0.0001 & $0.0011(=)$ & 0.0001 \\
\hline $\mathrm{HV}$ & 0.6613 & 0.0002 & $0.6612(=)$ & 0.0001 & $0.6614(=)$ & 0.0003 & $0.6612(=)$ & 0.0003 \\
\hline \multicolumn{9}{|l|}{ ZDT2 } \\
\hline IGD & 0.0038 & 0.0003 & $0.0037(=)$ & 0.0002 & $0.1955(+)$ & 0.2891 & $0.0041(=)$ & 0.0002 \\
\hline GD & 0.0008 & 0.0001 & $0.0009(=)$ & $0.0002(=)$ & $0.0005(-)$ & 0.0004 & $0.0009(=)$ & 0.0004 \\
\hline $\mathrm{HV}$ & 0.3287 & 0.0003 & $0.3284(=)$ & 0.0001 & $0.2245(+)$ & 0.1567 & $0.3283(=)$ & 0.0001 \\
\hline \multicolumn{9}{|l|}{ ZDT3 } \\
\hline IGD & 0.0100 & 0.0008 & $0.0111(=)$ & 0.0001 & $0.0049(-)$ & 0.0003 & $0.0059(-)$ & 0.0001 \\
\hline GD & 0.0040 & 0.0004 & $0.0041(=)$ & 0.0001 & $0.0050(+)$ & 0.0002 & $0.0044(+)$ & 0.0002 \\
\hline $\mathrm{HV}$ & 1.0383 & 0.0007 & $1.0376(+)$ & 0.0001 & $1.0417(=)$ & 0.0001 & $1.0387(=)$ & 0.0001 \\
\hline \multicolumn{9}{|l|}{ ZDT4 } \\
\hline IGD & 0.0042 & 0.0008 & $0.0042(=)$ & 0.0002 & $0.3547(+)$ & 0.4225 & $0.0045(+)$ & 0.0003 \\
\hline GD & 0.0683 & 0.1114 & $0.0021(-)$ & 0.0001 & $0.0015(-)$ & 0.0011 & $0.0032(-)$ & 0.0021 \\
\hline $\mathrm{HV}$ & 0.6609 & 0.0011 & $0.6611(=)$ & 0.0001 & $0.3830(+)$ & 0.3355 & $0.6605(=)$ & 0.0002 \\
\hline \multicolumn{9}{|l|}{ ZDT6 } \\
\hline IGD & 0.0037 & 0.0019 & $0.0039(=)$ & 0.0001 & $0.0028(=)$ & 0.0002 & $0.0038(=)$ & 0.0001 \\
\hline GD & 0.0051 & 0.0233 & $0.0008(-)$ & 0.0001 & $0.0011(-)$ & 0.0000 & $0.0031(-)$ & 0.0002 \\
\hline $\mathrm{HV}$ & 0.3226 & 0.0002 & $0.3221(=)$ & 0.0001 & $0.3217(=)$ & 0.0002 & $0.3221(=)$ & 0.0001 \\
\hline \multicolumn{9}{|l|}{ DTLZ1 } \\
\hline IGD & 0.0231 & 0.0009 & $0.0234(=)$ & 0.0001 & $0.0254(+)$ & 0.0013 & $0.0244(+)$ & 0.0002 \\
\hline GD & 0.0045 & 0.0013 & $0.0045(=)$ & 0.0001 & $0.0051(+)$ & 0.0002 & $0.0048(+)$ & 0.0001 \\
\hline $\mathrm{HV}$ & 0.9733 & 0.0053 & $0.9666(+)$ & 0.0010 & $0.9714(+)$ & 0.0004 & $0.9712(+)$ & 0.0001 \\
\hline \multicolumn{9}{|l|}{ DTLZ2 } \\
\hline IGD & 0.0611 & 0.0018 & $0.0619(=)$ & 0.0004 & $0.0674(+)$ & 0.0027 & $0.0621(+)$ & 0.0004 \\
\hline GD & 0.0034 & 0.0004 & $0.0034(=)$ & 0.0002 & $0.0078(+)$ & 0.0007 & $0.0045(+)$ & 0.0006 \\
\hline $\mathrm{HV}$ & 0.3910 & 0.0053 & $0.3802(+)$ & 0.0017 & $0.3824(+)$ & 0.0063 & $0.3865(+)$ & 0.0004 \\
\hline \multicolumn{9}{|l|}{ DTLZ3 } \\
\hline IGD & 0.0608 & 0.0018 & $0.0614(+)$ & 0.0002 & $0.0696(+)$ & 0.0036 & $0.0621(+)$ & 0.0014 \\
\hline GD & 0.0127 & 0.0005 & $0.0151(+)$ & 0.0004 & $0.0133(+)$ & 0.0006 & $0.0141(+)$ & 0.0008 \\
\hline $\mathrm{HV}$ & 0.3933 & 0.0072 & $0.3808(+)$ & 0.0013 & $0.3844(+)$ & 0.0053 & $0.3853(+)$ & 0.0007 \\
\hline \multicolumn{9}{|l|}{ DTLZ4 } \\
\hline IGD & 0.0615 & 0.0023 & $0.0627(+)$ & 0.0012 & $0.1162(+)$ & 0.2241 & $0.0620(+)$ & 0.0024 \\
\hline GD & 0.0136 & 0.0006 & $0.0168(+)$ & 0.0006 & $0.0180(+)$ & 0.0042 & $0.0164(+)$ & 0.0052 \\
\hline $\mathrm{HV}$ & 0.3923 & 0.0045 & $0.3840(+)$ & 0.0032 & $0.3626(+)$ & 0.0879 & $0.3867(+)$ & 0.0037 \\
\hline
\end{tabular}

"+" means that the proposed algorithm outperforms its competitor algorithm, "-" means that the proposed algorithm is outperformed by its competitor algorithm, and "=" means that the competitor algorithm has the same performance as the proposed algorithm. 
TABLE 9: The different patterns of investment of MVS-E model.

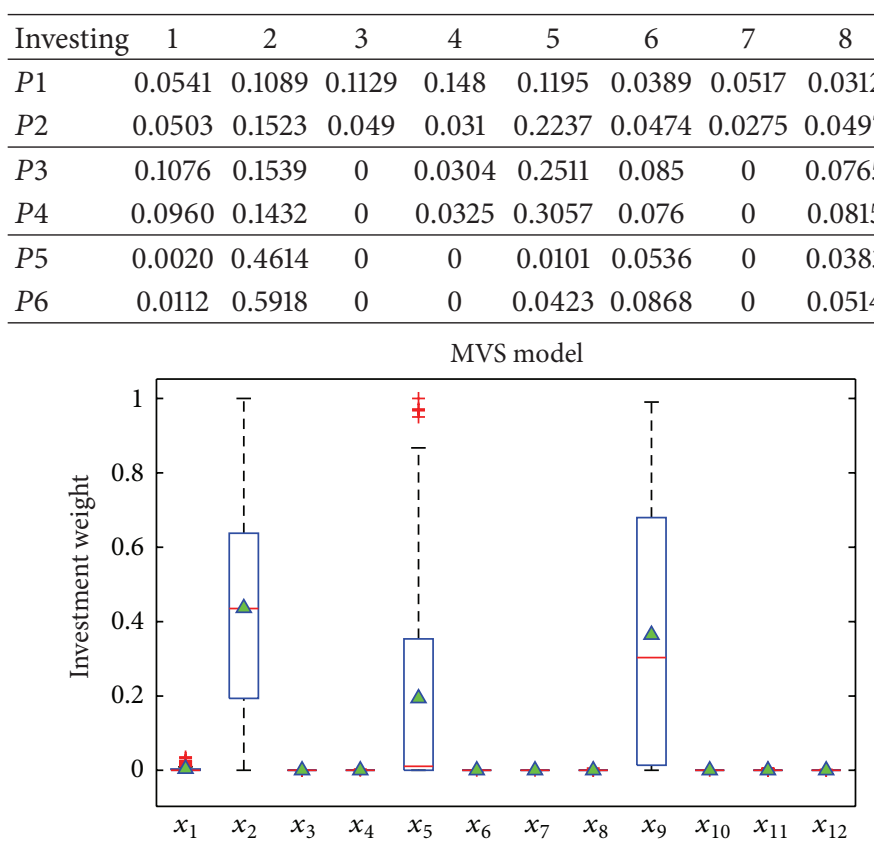

(a)

\begin{tabular}{cccccccc}
9 & 10 & 11 & 12 & Return & Risk & Skewness & Entropy \\
0.1538 & 0.0034 & 0.112 & 0.0656 & 0.0077 & 0.0023 & 0.0009 & 1.8332 \\
0.2652 & 0.0116 & 0.0499 & 0.0424 & 0.0076 & 0.0022 & 0.0008 & 1.3426 \\
\hline 0.2335 & 0 & 0 & 0.062 & 0.0053 & 0.0016 & 0.0005 & 0.5951 \\
0.1941 & 0 & 0 & 0.071 & 0.0052 & 0.0015 & 0.0004 & 0.5037 \\
0.4346 & 0 & 0 & 0 & 0.0034 & 0.0012 & 0.0003 & 0.2275 \\
0.2165 & 0 & 0 & 0 & 0.0032 & 0.0011 & 0.0002 & 0.0264
\end{tabular}

MVS-E model

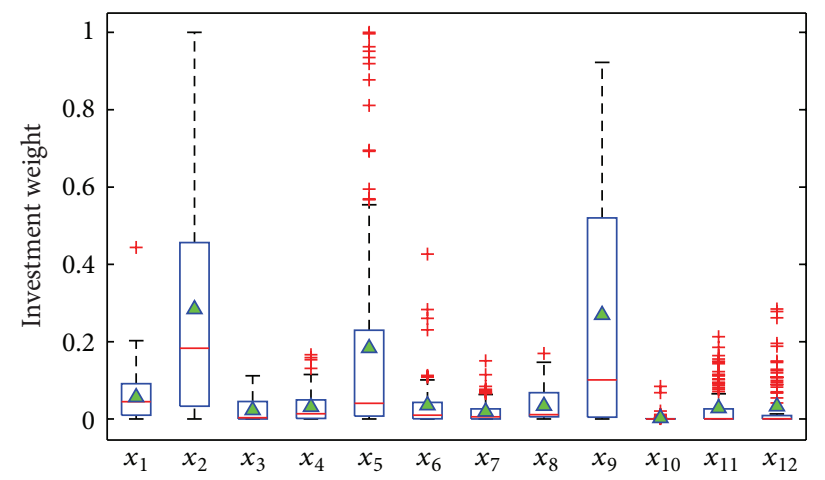

(b)

FIgure 4: (a) Portfolio distribution in PS of MVS model. (b) Portfolio distribution in PS of MVS-E model.

excessively allocates portfolio weights to a particular trading choice, but the portfolio weights of other assets are very small, even negligible. Figure 4(b) presents the optimal portfolio allocation solutions of the MVS-E model. The optimal portfolio weights increased to an average of 5.67\%, 2.3\%, 3.12\%, $3.54 \%, 1.92 \%, 3.44 \%, 0.26 \%, 2.81 \%$, and $3.28 \%$ corresponding to the $x_{1}, x_{3}, x_{4}, x_{6}, x_{7}, x_{8}, x_{10}, x_{11}$, and $x_{12}$, respectively, while the optimal portfolio weights exhibit a reduction to $28.41 \%, 18.34 \%$, and $26.91 \%$ in the $x_{2}, x_{5}$, and $x_{9}$, respectively. The inclusion of the fourth objective has not only allocated the Pareto-optimal portfolios more uniformly, but has also spread out the investment to more trading choices that will increase the diversification benefit.

Furthermore, Tables 7 and 9 show the specific sharing values for some of the Pareto-optimal portfolios of the final generation for MVS and MVS-E model. Here some representatives of the Pareto-optimal portfolios appear, corresponding to different patterns of investment. There are three different risk profiles which are associated with certain patterns of investment: risky $\{P 1, P 2\}$, medium risk $\{P 3, P 4\}$, and conservative $\{P 5, P 6\}$. The variability among the portfolios in each pattern may be small, but large among different patterns of investment.

As can be seen from Table 7, in three different patterns of investment, the vast majority of portfolio weights of PMV model extremely concentrated on the 9th asset, 5th asset, or 2 th asset. But the portfolio weights of other assets are very small, even negligible, which is a contradiction to the notion of diversification. It means that the MVS portfolios are quite concentrative. The very low diversity of the portfolios may result in loss while some of the invested assets experience unexpected gains.
From Table 9, MVS-E model can allocate more diversified portfolio weights to all the twelve assets; the larger the value of entropy function is, the more the diversified portfolio weights are. It is because adding the entropy function in MVS model enhances diversity of the portfolios. The diversified portfolio implies that the idiosyncratic risk can be decreased to zero as the assets included in the investment increase [36].

The results of numerical examples suggest that our proposed algorithm can significantly outperform MOEA/D and NSGA-II; in addition, we find that the MVS-E model can provide well-diversified Pareto-optimal solutions compared to the MVS model in the decision space. As a result, the MVS$\mathrm{E}$ model can give more and better alternative investment strategies compared to the MVS model. Through the above comparative analysis, we found that, by adding the entropy function in the MVS model, the corresponding improved MVS-E model can generate more diversity and uniformity Pareto-optimal portfolios. Moreover, the MVS-E model is able to provide more spread and diverse Pareto-optimal solutions in the PS; thus, this means that the MVS-E model performs well out of the MVS model.

5.5. Comparisons of the Proposed Algorithm with MOEA/D, NSGA-II, and D2MOPSO. D2MOPSO [37] is a multiobjective particle swarm optimizer (PSO) that incorporates the dominance concept with the decomposition approach. D2MOPSO uses the PSO framework to solve MOPs. It decomposes the MOP into a number of subproblems by a set of aggregation problems to determine the personal best of each particle and uses the Pareto dominance to select the leaders from the leader's archive to avoid premature convergence. In order to compare the proposed algorithm 
with MOEA/D, NSGA-II, and D2MOPSO, we used DTLZ1DTLZ4 [38], ZDT1-ZDT4, and ZDT6 [39] as the test problems. The population size of the proposed algorithm, MOEA/D, NSGA-II, and D2MOPSO is set to 105 for two and three objectives problems, respectively, and the maximal number of function evaluations is set to 100,000 for these nine test problems. In this paper, the following four performance metrics are used to evaluate the performance of the different algorithms quantitatively: generational distance (GD) [40], inverted generational distance (IGD) [40], and hypervolume indicator (HV) [33]. Wilcoxon Rank-Sum Test [41] is used in the sense of statistics to compare the mean IGD, GD, and HV of the compared algorithms. It tests whether the performance of the proposed algorithm on each test problem is better ("+"), same ("="), or worse ("-") than/as that of the compared algorithms at a significance level of 0.05 by a two-tailed test (Table 8).

\section{Conclusions}

In this paper, considering the six criteria, return, risk, skewness, transaction cost, liquidity, and diversification degree of portfolio, we propose two kinds of multiobjective fuzzy portfolio selection models based on new weighted moments of fuzzy variable. To enhance feasibility in the real world, we directly incorporate transaction costs and liquidity in the proposed models. The addition of entropy in the multiobjective portfolio model will help to avoid underdiversified portfolios (due to corner solutions) and to reduce the impact of the estimation risk. Furthermore, a multiobjective evolutionary algorithm based on decomposition of the objective space is designed to well maintain the diversity of obtained solutions. In order to achieve the goal, the objective space of the MOPs is decomposed into a number of subobjective spaces, and the obtained solutions are classed to make each subobjective space have a solution. For each subobjective space, if a new solution will replace its current solution whose objective vector locates in this subregion, the new solution must dominate the current solution and its objective vector locates in the subregion; if the objective vector of the current solution does not locate in this subregion, the new solution dominates the current solution or its objective vector locates in the subregion. In such a way, good population diversity can be achieved, which is essential for solving some MOPs. In addition, in order to improve the convergence of all obtained solutions, the crowding distance is used to calculate the fitness value of a solution for the selection operators, which can make dominated solutions more likely to be selected to generate new solutions. In order to demonstrate the effectiveness of the proposed algorithm, the performance of our algorithm is compared with the classic MOEA/D, NSGA-II, and D2MOPSO through some numerical examples. Simulation results show that our proposed algorithm is able to obtain better diversity and more evenly distributed Pareto front than the other three algorithms. Finally, the comparison analysis between the new weighted possibilistic MVS-E model and the weighted possibilistic MVS model is provided by numerical examples to illustrate the efficiency of the proposed approaches and the designed algorithm. The obtained results show that using the entropy in portfolio selection can diversify the allocation on various assets, while meeting the requirement of the different investors.

\section{Conflict of Interests}

The authors have declared that no conflict of interests exists.

\section{Acknowledgments}

This work was supported by National Natural Science Foundations of China (no. 61272119, no. 61402350) and the Fundamental Research Funds for the Central Universities (BDZ021430).

\section{References}

[1] H. Markowitz, "Portfolio selection," The Journal of Finance, vol. 7, no. 1, pp. 77-91, 1952.

[2] H. E. Leland, "Beyond mean-variance: performance measurement in a nonsymmetrical world," Financial Analysts Journal, vol. 55, no. 1, pp. 27-36, 1999.

[3] K. Kerstens, A. Mounir, and I. Van de Woestyne, "Geometric representation of the mean-variance-skewness portfolio frontier based upon the shortage function," European Journal of Operational Research, vol. 210, no. 1, pp. 81-94, 2011.

[4] F. D. Arditti and H. Levy, "Portfolio efficiency analysis in three moments: the multi-period case," The Journal of Finance, vol. 30, no. 3, pp. 797-809, 1975.

[5] J.-Y. Lai, "Shock-dependent conditional skewness in international aggregate stock markets," The Quarterly Review of Economics and Finance, vol. 52, no. 1, pp. 72-83, 2012.

[6] C. J. Adcock and K. Shutes, "An analysis of skewness and skewness persistence in three emerging markets," Emerging Markets Review, vol. 6, no. 4, pp. 396-418, 2005.

[7] P. K. Narayan and H. A. Ahmed, "Importance of skewness in decision making: evidence from the Indian stock exchange," Global Finance Journal, vol. 25, no. 3, pp. 260-269, 2014.

[8] R. Engle and A. Mistry, "Priced risk and asymmetric volatility in the cross section of skewness," Journal of Econometrics, vol. 182, no. 1, pp. 135-144, 2014.

[9] N. M. Pindoriya, S. N. Singh, and S. K. Singh, "Multi-objective mean-variance-skewness model for generation portfolio allocation in electricity markets," Electric Power Systems Research, vol. 80, no. 10, pp. 1314-1321, 2010.

[10] T. Eichner and A. Wagener, "Increases in skewness and threemoment preferences," Mathematical Social Sciences, vol. 61, no. 2, pp. 109-113, 2011.

[11] L. Yu, S. Y. Wang, and K. K. Lai, "Neural network-based meanvariance-skewness model for portfolio selection," Computers \& Operations Research, vol. 35, no. 1, pp. 34-46, 2008.

[12] R. Bhattacharyya, S. Kar, and D. D. Majumder, "Fuzzy meanvariance-skewness portfolio selection models by interval analysis," Computers \& Mathematics with Applications, vol. 61, no. 1, pp. 126-137, 2011.

[13] S. Barak, M. Abessi, and M. Modarres, "Fuzzy turnover rate chance constraints portfolio model," European Journal of Operational Research, vol. 228, no. 1, pp. 141-147, 2013.

[14] Y.-J. Liu, W.-G. Zhang, and W.-J. Xu, "Fuzzy multi-period portfolio selection optimization models using multiple criteria," Automatica, vol. 48, no. 12, pp. 3042-3053, 2012. 
[15] A. K. Bera and S. Y. Park, "Optimal portfolio diversification using the maximum entropy principle," Econometric Reviews, vol. 27, no. 4-6, pp. 484-512, 2008.

[16] Z. Chen and Y. Wang, "Two-sided coherent risk measures and their application in realistic portfolio optimization," Journal of Banking and Finance, vol. 32, no. 12, pp. 2667-2673, 2008.

[17] J.-R. Yu, W.-Y. Lee, and W. J. P. Chiou, "Diversified portfolios with different entropy measures," Applied Mathematics and Computation, vol. 241, pp. 47-63, 2014.

[18] P. Jana, T. K. Roy, and S. K. Mazumder, "Multi-objective possibilistic model for portfolio selection with transaction cost," Journal of Computational and Applied Mathematics, vol. 228, no. 1, pp. 188-196, 2009.

[19] X. Huang, "An entropy method for diversified fuzzy portfolio selection," International Journal of Fuzzy Systems, vol. 14, no. 1, pp. 160-165, 2012.

[20] K. Deb, A. Pratap, S. Agarwal, and T. Meyarivan, "A fast and elitist multi-objective genetic algorithm: NSGA-II," IEEE Transactions on Evolutionary Computation, vol. 6, no. 2, pp. 182197, 2002.

[21] Q. Zhang and H. Li, "MOEA/D: a multiobjective evolutionary algorithm based on decomposition," IEEE Transactions on Evolutionary Computation, vol. 11, no. 6, pp. 712-731, 2007.

[22] B. Soylu and M. Köksalan, "A favorable weight-based evolutionary algorithm for multiple criteria problems," IEEE Transactions on Evolutionary Computation, vol. 14, no. 2, pp. 191-205, 2010.

[23] E. C. Jara, "Multi-objective optimization by using evolutionary algorithms: the p-optimality criteria," IEEE Transactions on Evolutionary Computation, vol. 18, no. 2, pp. 167-179, 2014.

[24] C. Carlsson and R. Fuller, "On possibilistic mean value and variance of fuzzy numbers," Fuzzy Sets and Systems, vol. 122, no. 2, pp. 315-326, 2001.

[25] R. Fullér and P. Majlender, "On weighted possibilistic mean and variance of fuzzy numbers," Fuzzy Sets and Systems, vol. 136, no. 3, pp. 363-374, 2003.

[26] E. Pasha, B. Asady, and A. Sadidifar, "Weighted possibilistic variance and moments of fuzzy numbers," Journal of Applied Mathematics and Informatics, vol. 26, no. 5-6, pp. 1169-1183, 2008.

[27] D. A. van Veldhuizen, Multiobjective evolutionary algorithms: classifications, analyses, and new innovations [Ph.D. thesis], Department of Electrical and Computer Engineering, Graduate School of Engineering, Air Force Institute of Technology, Wright-Patterson AFB, Ohio, USA, 1999.

[28] R. Goetschel Jr. and W. Voxman, "Elementary fuzzy calculus," Fuzzy Sets and Systems, vol. 18, no. 1, pp. 31-43, 1986.

[29] E. Vercher, J. D. Bermúdez, and J. V. Segura, "Fuzzy portfolio optimization under downside risk measures," Fuzzy Sets and Systems, vol. 158, no. 7, pp. 769-782, 2007.

[30] K. Deb, Multi-Objective Optimization Using Evolutionary Algorithms, John Wiley \& Sons, New York, NY, USA, 2001.

[31] R. Storn and K. Price, "Differential evolution-a simple and efficient heuristic for global optimization over continuous spaces," Journal of Global Optimization, vol. 11, no. 4, pp. 341359, 1997.

[32] E. Zitzler, L. Thiele, M. Laumanns, C. M. Fonseca, and V. G. Da Fonseca, "Performance assessment of multiobjective optimizers: an analysis and review," IEEE Transactions on Evolutionary Computation, vol. 7, no. 2, pp. 117-132, 2003.

[33] K. Deb, A. Sinha, and S. Kukkonen, "Multi-objective test problems, linkages, and evolutionary methodologies," in Proceedings of the 8th Annual Genetic and Evolutionary Computation Conference (GECCO '06), pp. 1141-1148, Seattle, Wash, USA, July 2006.

[34] A. Biglova, S. Ortobelli, S. Rachev, and S. Stoyanov, "Different approaches to risk estimation in portfolio theory," The Journal of Portfolio Management, vol. 31, no. 1, pp. 103-112, 2004.

[35] V. Zakamouline and S. Koekebakker, "Portfolio performance evaluation with generalized Sharpe ratios: beyond the mean and variance," Journal of Banking \& Finance, vol. 33, no. 7, pp. 12421254, 2009.

[36] A. Y. Popkov, "Entropy model of the investment portfolio," Automation and Remote Control, vol. 67, pp. 1518-1528, 2006.

[37] N. Al Moubayed, A. Petrovski, and J. McCall, " $D^{2} M O P S O$ : MOPSO based on decomposition and dominance with archiving using crowding distance in objective and solution spaces," Evolutionary Computation, vol. 22, no. 1, pp. 47-78, 2014.

[38] K. Deb, L. Thiele, M. Laumanns, and E. Zitzler, "Scalable multiobjective optimization test problems," in Proceedings of the Congress on Evolutionary Computation (CEC '02), pp. 825-830, May 2002.

[39] E. Zitzler, K. Deb, and L. Thiele, "Comparison of multiobjective evolutionary algorithms: empirical results." Evolutionary computation, vol. 8, no. 2, pp. 173-195, 2000.

[40] C. A. C. Coello and N. C. Cortés, "Solving multiobjective optimization problems using an artificial immune system," Genetic Programming and Evolvable Machines, vol. 6, no. 2, pp. 163-190, 2005.

[41] S. Robert, J. Torrie, and D. Dickey, Principles and Procedures of Statistics: A Biometrical Approach, McGraw-Hill, New York, NY, USA, 1997. 


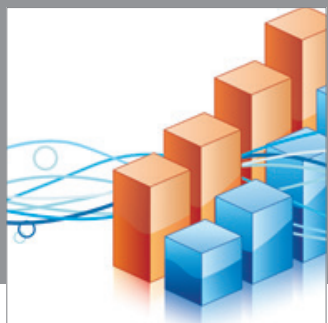

Advances in

Operations Research

mansans

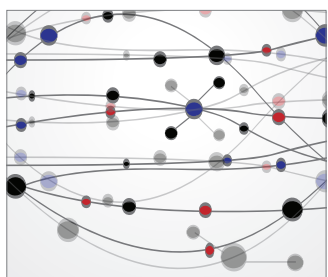

The Scientific World Journal
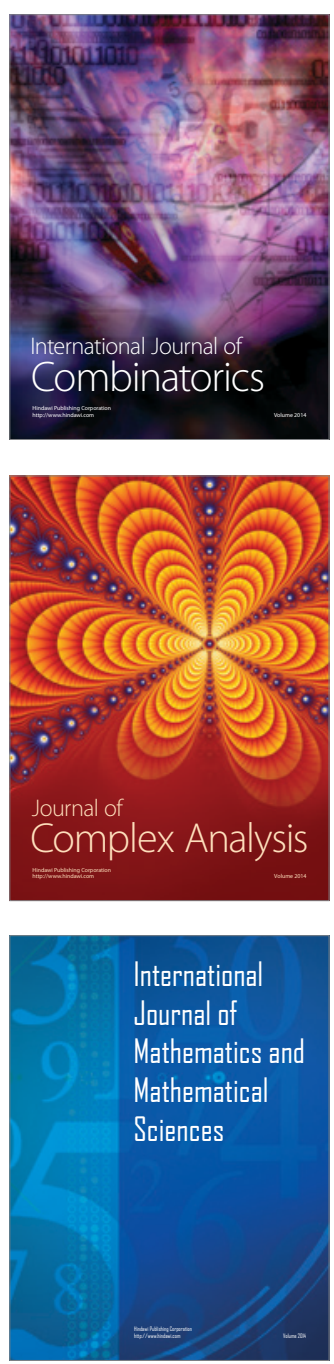
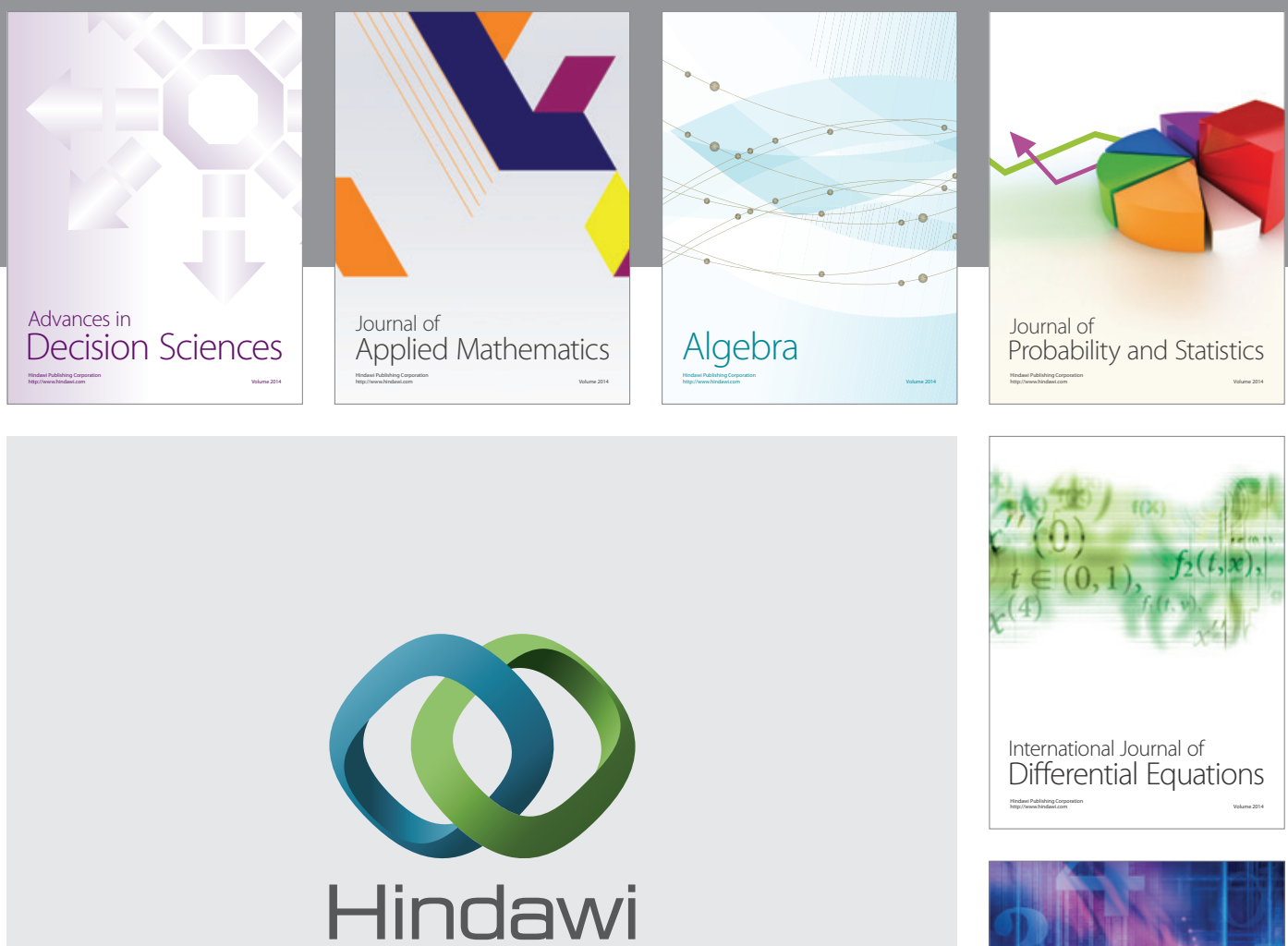

Submit your manuscripts at http://www.hindawi.com
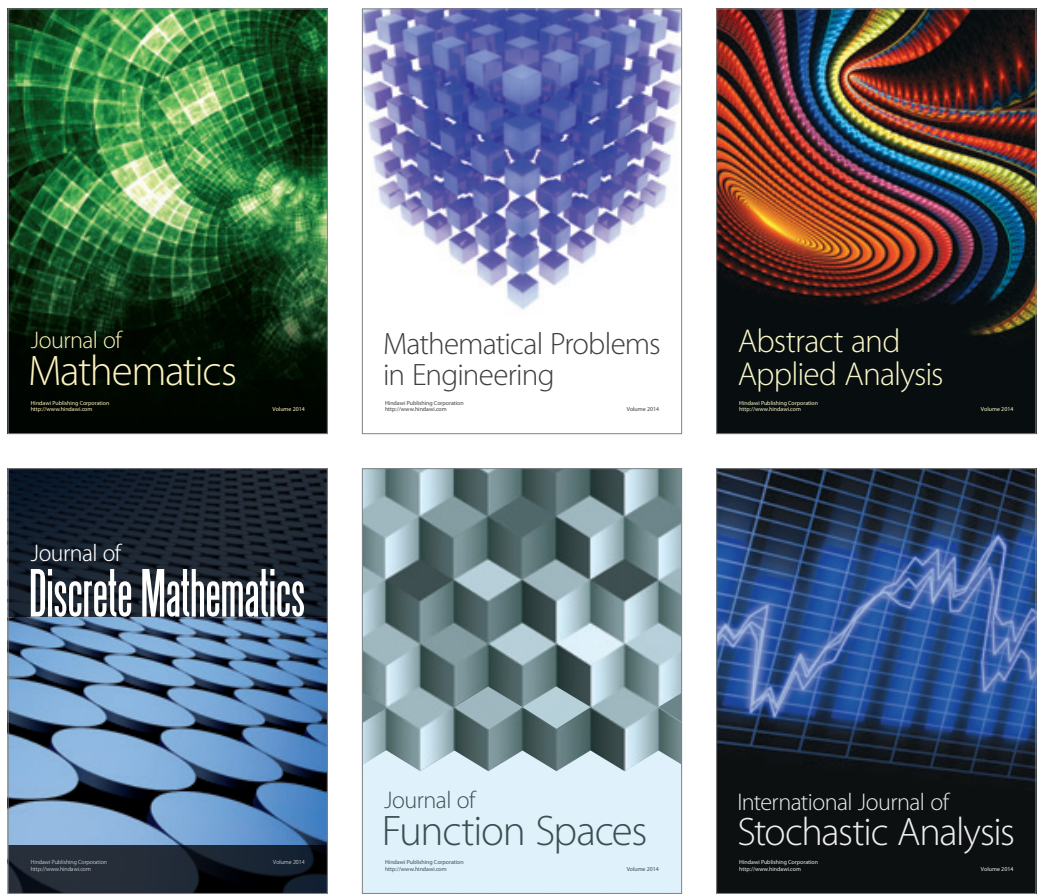

Journal of

Function Spaces

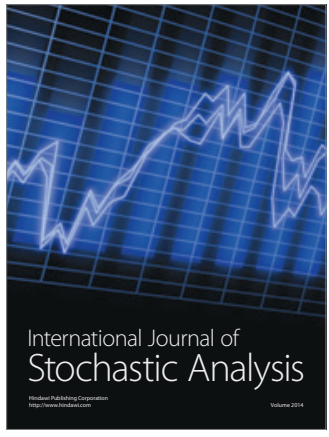

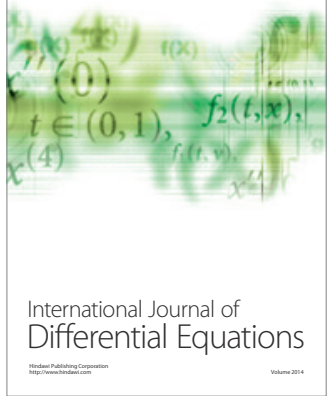
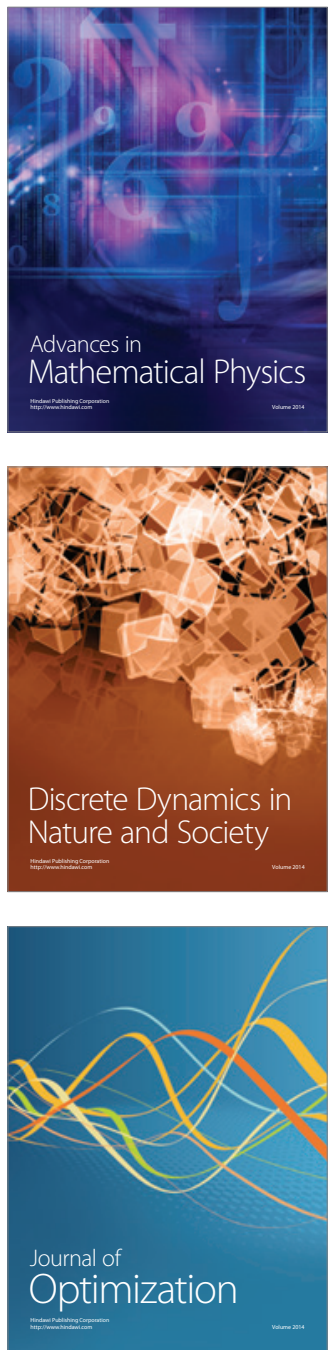